\title{
Consistency between Lüscher's finite volume method and HAL QCD method for two-baryon systems in lattice QCD
}

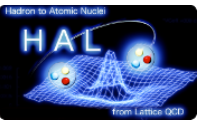

\section{The HAL QCD collaboration}

Takumi Iritani, ${ }^{a}$ Sinya Aoki, ${ }^{b, c}$ Takumi Doi, ${ }^{a, d}$ Tetsuo Hatsuda, ${ }^{a, d}$ Yoichi Ikeda, ${ }^{e}$ Takashi Inoue, ${ }^{f}$ Noriyoshi Ishii, ${ }^{e}$ Hidekatsu Nemura ${ }^{e}$ and Kenji Sasaki ${ }^{b}$

${ }^{a}$ RIKEN Nishina Center, Wako 351-0198, Japan

${ }^{b}$ Center for Gravitational Physics, Yukawa Institute for Theoretical Physics, Kyoto University, Kitashirakawa Oiwakecho, Sakyo-ku, Kyoto 606-8502, Japan

${ }^{c}$ Center for Computational Sciences, University of Tsukuba, Tsukuba 305-8577, Japan

${ }^{d}$ RIKEN Interdisciplinary Theoretical and Mathematical Science Program (iTHEMS),

Wako 351-0198, Japan

${ }^{e}$ Research Center for Nuclear Physics (RCNP), Osaka University, Osaka 567-0047, Japan

${ }^{f}$ Nihon University, College of Bioresource Sciences, Kanagawa 252-0880, Japan

E-mail: takumi.iritani@riken.jp, saoki@yukawa.kyoto-u.ac.jp,

doi@ribf.riken.jp, thatsuda@riken.jp, yikeda@rcnp.osaka-u.ac.jp,

inoue.takashi@nihon-u.ac.jp, ishiin@rcnp.osaka-u.ac.jp,

hidekatsu.nemura@rcnp.osaka-u.ac.jp, kenjis@yukawa.kyoto-u.ac.jp

ABSTRACT: There exist two methods to study two-baryon systems in lattice QCD: the direct method which extracts eigenenergies from the plateaux of the temporal correlation function and the HAL QCD method which extracts observables from the non-local potential associated with the tempo-spatial correlation function. Although the two methods should give the same results theoretically, there have been reported qualitative difference for observables from lattice QCD simulations. Recently, we pointed out in $[1,2]$ that the separation of the ground state from the excited states is crucial to obtain sensible results in the former, while both states provide useful signals for observables in the latter. In this paper, we identify the contribution of each state in the direct method by decomposing the two-baryon correlation functions into the finite-volume eigenmodes obtained from the HAL QCD method. As in our previous studies, we consider the $\Xi \Xi$ system in the ${ }^{1} \mathrm{~S}_{0}$ channel 
at $m_{\pi}=0.51 \mathrm{GeV}$ in (2+1)-flavor lattice QCD using the wall and smeared quark sources with spatial extents, $L a=3.6,4.3,5.8 \mathrm{fm}$. We demonstrate that the "pseudo-plateau" at early time slices $(t=1 \sim 2 \mathrm{fm}$ ) from the smeared source in the direct method indeed originates from the contamination of the excited states, and the plateau with the ground state saturation is realized only at $t>5 \sim 15 \mathrm{fm}$ corresponding to the inverse of the lowest excitation energy. We also demonstrate that the two-baryon operator can be optimized by utilizing the finite-volume eigenmodes, so that (i) the finite-volume energy spectra from the HAL QCD method agree with those from the temporal correlation function with the optimized operators and (ii) the correct finite-volume spectra would be accessed in the direct method only if highly optimized operators are employed. Thus we conclude that the long-standing issue on the consistency between Lüscher's finite volume method and the HAL QCD method for two baryons is now resolved at least for this particular system considered here: they are consistent with each other quantitatively only if the excited contamination is properly removed in the former.

KEYwords: Lattice QCD, Lattice Quantum Field Theory

ARXIV EPRINT: 1812.08539 


\section{Contents}

1 Introduction 1

2 Methods and Lattice setup 3

2.1 Direct method 3

2.2 HAL QCD method 4

2.3 Lattice setup 6

3 Summary of previous studies $\quad 6$

3.1 Operator dependence of the plateaux in the direct method 6

3.2 Normality check 8

3.3 Source-operator dependence and derivative expansion of the potentials 8

4 Anatomy: excited state contaminations in the effective energy shifts 10

$\begin{array}{lll}4.1 & \text { Eigenvalues and eigenfunctions in the finite box } & 11\end{array}$

$\begin{array}{ll}4.2 & \text { Decomposition of the } R \text {-correlator via eigenfunctions } \\ & 12\end{array}$

$\begin{array}{lll}4.3 & \text { Reconstruction of the effective energy shift } & 14\end{array}$

$\begin{array}{lll}4.4 & \text { Projection with improved sink operator } & 17\end{array}$

$\begin{array}{llr}5 & \text { Summary } & 19\end{array}$

A The finite volume spectra from the $\mathrm{N}^{2} \mathrm{LO}$ potential 20

$\begin{array}{ll}\text { B Eigenfunctions on various volumes } & 21\end{array}$

C Reconstruction of the $R$-correlator $\quad 21$

D Reconstructed effective energy shifts $\quad 23$

$\begin{array}{lll}\text { D.1 The results on various volumes } & 23\end{array}$

D.2 Contributions from excited states to the effective energy shifts 23

E Effective energy shifts from the improved sink operator based on $\begin{array}{ll}\text { eigenfunctions } & 26\end{array}$

\section{Introduction}

The interactions between two baryons have been studied by two methods in lattice QCD. The first one is the direct method [3-5], which extracts the eigenenergies of the ground and/or the excited states from the temporal correlations of two-baryon systems. The binding energies and scattering phase shifts are calculated from eigenenergies using Lüscher's finite volume formula [6-8]. The second one is the HAL QCD method [9-12], which derives the energy-independent non-local kernel (called the "potential" in the literature) from the 
tempo-spatial correlations of two baryons. Then the binding energies and phase shifts in the infinite volume are calculated by using the Schrödinger-type equation with the kernel as the potential, which has field theoretical derivation on the basis of the reduction formula for composite operators. Both methods rely on the asymptotic behavior of the Nambu-Bethe-Salpeter (NBS) wave function, and should in principle give the same results for observables $[10,12,13]$. In practice, however, the current numerical results for twonucleon $(N N)$ systems seem to be inconsistent with each other: for heavy pion masses $\left(m_{\pi}>0.3 \mathrm{GeV}\right)$, both dineutron $\left({ }^{1} \mathrm{~S}_{0}\right)$ and deuteron $\left({ }^{3} \mathrm{~S}_{1}\right)$ are claimed to be bound in the direct method, while those are unbound in the HAL QCD method. Also, the discrepancy is ubiquitous in two-baryon systems: although both methods indicate a bound H-dibaryon in the $\mathrm{SU}(3)$ flavor limit at $m_{\pi}=m_{K} \simeq 0.8 \mathrm{GeV}$, the binding energy is $74.6(4.7) \mathrm{MeV}$ in the direct method [14], while it is $37.8(5.2) \mathrm{MeV}$ in the HAL QCD method [15]. ${ }^{1}$

In a series of recent papers $[1,2,17-20]$, we have carefully examined the systematic uncertainties in both methods. The difficulty of two-baryon systems compared to a single baryon originates from the existence of elastic scattering states. Their typical excitation energies $\delta E$ are one to two orders of magnitude smaller than $\mathcal{O}\left(\Lambda_{\mathrm{QCD}}\right)$, so that one needs to probe large Euclidean time $t \gtrsim(\delta E)^{-1}$ to extract the genuine signal of the ground state in the direct method. However, the statistical fluctuation increases exponentially in $t$ as well as the baryon number $A$ for multi-baryon systems as proved in [21, 22]. This practically prevents one to identify the signal of the ground state in the naive analysis of the temporal correlation of two baryons.

Moreover, our extensive studies [1,2] showed that a commonly employed procedure in the direct method to identify plateaux at early time slices, $t \ll(\delta E)^{-1}$, suffers from uncontrolled systematic errors from the excited state contaminations, since pseudo-plateaux ${ }^{2}$ easily appear at early time slices. The typical symptoms of such systematics in the previous studies were explicitly exposed by the normality check $^{3}$ based on Lüscher's finite volume formula [8] and the analytic properties of the $S$-matrix [2].

As far as the HAL QCD method is concerned, the time-dependent formalism [11] is free from the problem of the ground state saturation, since the energy-independent potential is extracted from the spatial and temporal correlations with the information of both the ground and excited states associated with the elastic scattering. While in practical calculations there appears a systematic uncertainty associated with the truncation of the derivative expansion for the non-locality of the potential, the derivative expansion is found to be well converged at low energies $[20,24]$. Other systematic uncertainties such as the contaminations from the inelastic states and the finite volume effect for the potential are also shown to be well under control [20].

In this paper, we reveal the origin of the inconsistency between the direct method and the HAL QCD method, by explicitly evaluating the magnitude of the excited states in the

\footnotetext{
${ }^{1}$ Recently, there appears another study [16] using the direct method, which indicates the dineutron is unbound while the H-dibaryon is bound $19(10) \mathrm{MeV}$ at $m_{\pi}=m_{K}=0.96 \mathrm{GeV}$.

${ }^{2}$ In refs. $[1,2]$, they are called "fake plateaux" or "mirages" of the plateau of the ground state.

${ }^{3}$ In ref. [2], it is called "sanity check", a common terminology in computer science for a simple/quick test [23].
} 
temporal correlation functions. We focus on the $\Xi \Xi$ system in the ${ }^{1} \mathrm{~S}_{0}$ channel, which is a most convenient channel to obtain insights into the $N N$ systems, since it belongs to the same $\mathrm{SU}(3)$ flavor multiplet as $N N\left({ }^{1} S_{0}\right)$ but has much better statistical signals. Detailed studies in this channel were already performed with the direct method [1] as well as the HAL QCD method [20] in (2+1) flavor lattice QCD at $m_{\pi}=0.51 \mathrm{GeV}$ and $m_{K}=0.62 \mathrm{GeV}$, so that the main purpose of this paper is to present an in-depth analysis by combining both results: in particular, the excited state contaminations in the temporal correlation functions are quantitatively evaluated by decomposing them in terms of the finite-volume eigenmodes of Hamiltonian with the HAL QCD potential (the HAL QCD Hamiltonian).

We show how the pseudo-plateau actually appears at early time slices and also predict the time slice at which the ground state saturation is achieved. Moreover, we establish a consistency between the direct method and the HAL QCD method, by demonstrating that temporal correlation functions constructed from the optimized two-baryon operators by the eigenmode of the HAL QCD Hamiltonian show the plateaux with the values consistent with the eigenenergies at early time slices.

This paper is organized as follows. In section 2, we introduce the theoretical framework of the direct method and the HAL QCD method, and present the numerical setup of the lattice QCD calculation. In section 3, we recapitulate the previous analysis on the direct method [1] as well as on the HAL QCD method [20]. In section 4, we decompose the correlation functions into the eigenmodes of the HAL QCD Hamiltonian. The anatomy of the excited state contaminations in the direct method is presented. We also demonstrate that eigenfunctions can be used to optimize two baryon-operators. The consistency between the temporal correlations with the optimized operators and the HAL QCD method is established. Section 5 is devoted to the conclusion. In appendix A, we check how the nextto-next-leading order $\left(\mathrm{N}^{2} \mathrm{LO}\right)$ analysis for the HAL QCD potential affects the finite volume spectra. In appendix B, we collect eigenfunctions of the HAL QCD Hamiltonian on various volumes. In appendix $\mathrm{C}$, we study the reconstruction of the $R$-correlator from the elastic states. In appendix D, we collect the results for the reconstruction of the effective energy shifts. In appendix E, we show the effective energy shifts from the optimized operators on various volumes. We note that a preliminary account of this study was reported in refs. $[17,19]$.

\section{Methods and Lattice setup}

In this section, we briefly summarize the direct method and the HAL QCD method for two-baryon systems, together with the lattice setup used in this paper.

\subsection{Direct method}

In the direct method for two-baryon systems, the energy eigenvalues (on a finite volume) are measured from the temporal correlation of the two-baryon operator, $\mathcal{J}_{B B}^{\text {sink,src }}(t)$, as

$$
C_{\mathrm{BB}}(t) \equiv\left\langle 0\left|\mathcal{J}_{B B}^{\operatorname{sink}}(t) \overline{\mathcal{J}}_{B B}^{\mathrm{src}}(0)\right| 0\right\rangle=\sum_{n} Z_{n} e^{-W_{n} t}+\cdots
$$


where $W_{n}$ is the energy of $n$-th two-baryon elastic state and the ellipsis denotes the inelastic contributions. In order to obtain the energy shifts $\Delta E_{n} \equiv W_{n}-2 m_{B}$ with $m_{B}$ being the single baryon mass, one often uses the ratio of the temporal correlation function of two(one-) baryon system $C_{\mathrm{BB}}(t)\left(C_{\mathrm{B}}(t)\right)$ as

$$
R(t) \equiv \frac{C_{\mathrm{BB}}(t)}{\left\{C_{\mathrm{B}}(t)\right\}^{2}}, \quad C_{B}(t)=Z_{B} e^{-m_{B} t}+\cdots,
$$

to reduce the statistical uncertainties as well as some systematics thanks to the correlations between $C_{\mathrm{BB}}(t)$ and $C_{\mathrm{B}}(t)$. The energy shift of the ground state can be obtained from the plateau value of the effective energy shift defined by

$$
\Delta E_{\mathrm{eff}}(t) \equiv \frac{1}{a} \log \frac{R(t)}{R(t+a)}
$$

with $a$ being the lattice spacing. Here $t$ needs to be sufficiently large compared to the inverse of the excitation energy.

Once the energy shift of the ground (or excited) state on a finite volume is obtained, one can calculate the scattering phase shift in the infinite volume at that energy, $\delta_{0}(k)$, via Lüscher's finite volume formula [8],

$$
k \cot \delta_{0}(k)=\frac{1}{\pi(L a)} \sum_{\vec{n} \in \mathbf{Z}^{3}} \frac{1}{\vec{n}^{2}-q^{2}}, \quad q=\frac{k(L a)}{2 \pi},
$$

where we consider the S-wave scattering for simplicity, $k$ is defined through $W_{n}=2 \sqrt{m_{B}^{2}+k^{2}}$ and $L$ is the number of the spatial site of the lattice box. If the energy shift $\Delta E_{n}$ is negative, the analytic continuation of the above formula to $k^{2}<0$ is understood. The state with a negative energy shift in the infinite volume limit corresponds to a bound state.

As noted before, the origin of the difficulty of two-baryon systems is the existence of elastic scattering states. Since the typical excitation energy of such states is $(2 \pi)^{2} /\left((L a)^{2} m_{B}\right)$, the ground state saturation requires extremely large $t$, e.g., $t \gtrsim \mathcal{O}(4) \mathrm{fm}$ at $L a=4 \mathrm{fm}$ and $m_{B}=2 \mathrm{GeV}$, where the bad signal-to-noise ratio makes it practically impossible to obtain signals. In the literature of the direct method [3-5], however, one extracted the energy shift for the ground state from the plateau-like behavior of the effective energy shift at early time slices, $t \sim \mathcal{O}(1)$ fm instead, assuming that the ground state saturation is achieved.

\subsection{HAL QCD method}

In the HAL QCD method, the energy-independent non-local potential $U\left(\vec{r}, \overrightarrow{r^{\prime}}\right)$ is defined from

$$
\left(E_{k}-H_{0}\right) \psi^{W}(\vec{r})=\int d \overrightarrow{r^{\prime}} U\left(\vec{r}, \overrightarrow{r^{\prime}}\right) \psi^{W}\left(\overrightarrow{r^{\prime}}\right),
$$

with the Nambu-Bethe-Salpeter (NBS) wave function $[9,10]$,

$$
\psi^{W}(\vec{r})=\left\langle 0\left|T\left\{\sum_{\vec{x}} B(\vec{x}+\vec{r}, 0) B(\vec{x}, 0)\right\}\right| 2 B, W\right\rangle .
$$


Here $|2 B, W\rangle$ is the QCD eigenstate for two baryons with the eigenenergy $W=2 \sqrt{m_{B}^{2}+k^{2}}$ in the center of mass system, $B(\vec{x}, t)$ is a single baryon operator, $E_{k}=k^{2} /(2 \mu)$, and $H_{0}=-\nabla^{2} /(2 \mu)$ with $\mu=m_{B} / 2$ being the reduced mass. Eq. (2.5) has field theoretical derivation on the basis of the Nishijima-Zimmermann-Haag reduction formula for composite operators [25-27]. Below the inelastic threshold $W_{\mathrm{th}}$, the potential $U\left(\vec{r}, \overrightarrow{r^{\prime}}\right)$ is shown to be faithful to the phase shifts, which are encoded in the behaviors of the NBS wave functions at large $r$.

The four-point correlation function of the two-baryon system $F(\vec{r}, t)$ is given by

$$
\begin{aligned}
F(\vec{r}, t) & \equiv\left\langle 0\left|T\left\{\sum_{\vec{x}} B(\vec{x}+\vec{r}, t) B(\vec{x}, t) \overline{\mathcal{J}}_{B B}^{\mathrm{sc}}(0)\right\}\right| 0\right\rangle \\
& =\left\langle 0\left|T\left\{\sum_{\vec{x}} B(\vec{x}+\vec{r}, t) B(\vec{x}, t)\right\} \sum_{n}\right| 2 B, W_{n}\right\rangle\left\langle 2 B, W_{n}\left|\overline{\mathcal{J}}_{B B}^{\mathrm{src}}(0)\right| 0\right\rangle+\cdots \\
& =\sum_{n} A_{n} \psi^{W_{n}}(\vec{r}) e^{-W_{n} t}+\cdots,
\end{aligned}
$$

where $A_{n} \equiv\left\langle 2 B, W_{n}\left|\overline{\mathcal{J}}_{B B}^{\mathrm{src}}(0)\right| 0\right\rangle$ is the overlap factor and the ellipsis represents the inelastic contributions.

In the time-dependent HAL QCD method $[11,12]$, the potential is extracted directly from the so-called $R$-correlator as

$$
\left[-H_{0}-\frac{\partial}{\partial t}+\frac{1}{4 m_{B}} \frac{\partial^{2}}{\partial t^{2}}\right] R(\vec{r}, t)=\int d \overrightarrow{r^{\prime}} U\left(\vec{r}, \overrightarrow{r^{\prime}}\right) R\left(\overrightarrow{r^{\prime}}, t\right)
$$

where

$$
R(\vec{r}, t) \equiv \frac{F(\vec{r}, t)}{\left\{C_{B}(t)\right\}^{2}}=\sum_{n} \frac{A_{n}}{Z_{B}^{2}} \psi^{W_{n}}(\vec{r}) e^{-\left(W_{n}-2 m_{B}\right) t}+\cdots,
$$

with the ellipsis being the inelastic contributions. Eq. (2.9) requires neither the ground state saturation nor the determination of individual eigenenergy $W_{n}$ and eigenfunction $\psi^{W_{n}}(\vec{r})$, as all elastic states can be used to extract the energy-independent potential. Therefore, compared with the direct method, the condition required for the reliable calculation is much more relaxed in the time-dependent HAL QCD method as $t \gtrsim \mathcal{O}\left(\Lambda_{\mathrm{QCD}}^{-1}\right) \sim \mathcal{O}(1) \mathrm{fm}$, where $R(\vec{r}, t)$ is saturated by the contributions from elastic states ("the elastic state saturation").

In practice, we expand the non-local potential in terms of derivatives as $U\left(\vec{r}, \overrightarrow{r^{\prime}}\right)=$ $\sum_{n} V_{n}(\vec{r}) \nabla^{n} \delta\left(\vec{r}-\overrightarrow{r^{\prime}}\right)$. The leading order $(\mathrm{LO})$ approximation gives $U\left(\vec{r}, \overrightarrow{r^{\prime}}\right) \simeq V_{0}^{\mathrm{LO}}(r) \delta\left(\vec{r}-\vec{r}^{\prime}\right)$, which can be determined as

$$
V_{0}^{\mathrm{LO}}(\vec{r})=-\frac{H_{0} R(\vec{r}, t)}{R(\vec{r}, t)}-\frac{(\partial / \partial t) R(\vec{r}, t)}{R(\vec{r}, t)}+\frac{1}{4 m_{B}} \frac{(\partial / \partial t)^{2} R(\vec{r}, t)}{R(\vec{r}, t)} .
$$

We can also examine the effect of higher order contributions to observables such as the scattering phase shifts: in this paper, we present the study on the correction to the LO potential for the spin-singlet channel at the next-to-next-leading order $\left(\mathrm{N}^{2} \mathrm{LO}\right)$ as

$$
U\left(\vec{r}, \overrightarrow{r^{\prime}}\right) \simeq\left\{V_{0}^{\mathrm{N}^{2} \mathrm{LO}}(\vec{r})+V_{2}^{\mathrm{N}^{2} \mathrm{LO}}(\vec{r}) \nabla^{2}\right\} \delta\left(\vec{r}-\overrightarrow{r^{\prime}}\right) .
$$




\begin{tabular}{|c|c|c|cc|c|}
\hline volume & $L a$ & \# of conf. & \# of smeared sources & $(A, B)$ & \# of wall sources \\
\hline $40^{3} \times 48$ & $3.6 \mathrm{fm}$ & 207 & 512 & $(0.8,0.22)$ & 48 \\
$48^{3} \times 48$ & $4.3 \mathrm{fm}$ & 200 & $4 \times 384$ & $(0.8,0.23)$ & $4 \times 48$ \\
$64^{3} \times 64$ & $5.8 \mathrm{fm}$ & 327 & $1 \times 256$ & $(0.8,0.23)$ & $4 \times 64$ \\
\hline
\end{tabular}

Table 1. Simulation parameters. The rotational symmetry for isotropic lattice is used to increase statistics.

\subsection{Lattice setup}

Numerical data in previous literature $[1,20]$ and in this paper are obtained from the $(2+1)$-flavor lattice QCD ensembles, generated in ref. [28] with the Iwasaki gauge action and nonperturbatively $\mathcal{O}(a)$-improved Wilson quark action at the lattice spacing $a=0.08995(40) \mathrm{fm}\left(a^{-1}=2.194(10) \mathrm{GeV}\right)$. In the present paper, we make use of gauge ensembles on three lattice volumes, $L^{3} \times T=40^{3} \times 48,48^{3} \times 48$, and $64^{3} \times 64$, with heavy up and down quark masses and the physical strange quark masses, corresponding to $m_{\pi}=0.51 \mathrm{GeV}, m_{K}=0.62 \mathrm{GeV}, m_{N}=1.32 \mathrm{GeV}$ and $m_{\Xi}=1.46 \mathrm{GeV}$.

We employ two different quark sources with the Coulomb gauge fixing, the wall source, $q^{\text {wall }}(t)=\sum_{\vec{y}} q(\vec{y}, t)$, mainly used in the HAL QCD method, and the smeared source, $q^{\text {smear }}(\vec{x}, t)=\sum_{\vec{y}} f(|\vec{x}-\vec{y}|) q(\vec{y}, t)$, often used in the direct method. For the smearing function, we take $f(r) \equiv\left\{A e^{-B r}, 1,0\right\}$ for $\{0<r<(L-1) / 2, r=0,(L-1) / 2 \leq r\}$, respectively, as in ref. [28], and the center of the smeared source is same for all six quarks (i.e., zero displacement between two baryons), as has been employed in all previous studies in the direct method claiming the existence of the $N N$ bound states for heavy quark masses $[3-5] .{ }^{4}$ For both sources, the point-sink operator for each baryon is employed in this study. A number of configurations and other parameters are summarized in table 1. The correlation functions are calculated by the unified contraction algorithm (UCA) [29] and the statistical errors are evaluated by the jack-knife method. For more details on the simulation setup, see ref. [1].

In this paper, we focus on $\Xi \Xi\left({ }^{1} \mathrm{~S}_{0}\right)$ system, which belongs to the same $\mathbf{2 7}$ representation as $N N\left({ }^{1} \mathrm{~S}_{0}\right)$ in the flavor $\mathrm{SU}(3)$ transformation, but has much better signal-to-noise ratio than $N N$ as the system contains four strange quarks. We use the relativistic interpolating operator [1] for $\Xi$, given by

$$
\Xi_{\alpha}^{0}=\varepsilon_{a b c}\left(s^{a T} C \gamma_{5} u^{b}\right) s_{\alpha}^{c}, \quad \Xi_{\alpha}^{-}=\varepsilon_{a b c}\left(s^{a T} C \gamma_{5} d^{b}\right) s_{\alpha}^{c},
$$

where $C=\gamma_{4} \gamma_{2}$ is the charge conjugation matrix, $\alpha$ and $a, b, c$ are the spinor and color indices, respectively.

\section{Summary of previous studies}

\subsection{Operator dependence of the plateaux in the direct method}

In ref. [1], we pointed out that the plateau-like behaviors at $t \simeq 1 \mathrm{fm}$ in the direct method depend on sources or sink operators. For example, figure 1 (left) shows the source operator

\footnotetext{
${ }^{4}$ Ref. [5] uses a non-zero displaced operator as well.
} 

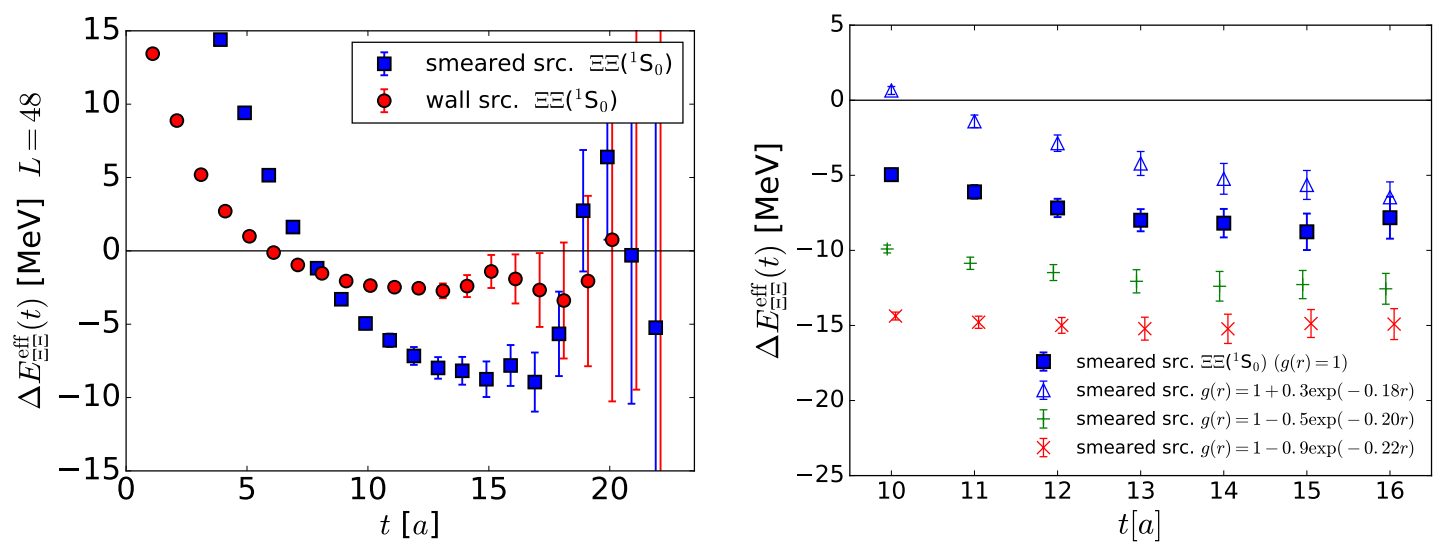

Figure 1. (Left) The source operator dependence of the effective energy shift $\Delta E_{\text {eff }}(t)$ for $\Xi \Xi\left({ }^{1} \mathrm{~S}_{0}\right)$ using the wall source (red circles) and the smeared source (blue squares) for $L=48$ [1]. (Right) The sink operator dependence of the same quantity with the smeared source [1].

dependence of the effective energy shift $\Delta E_{\text {eff }}(t)$ in eq. (2.3) for the $\Xi \Xi\left({ }^{1} \mathrm{~S}_{0}\right)$ on $L=48$, where $\mathcal{J}_{B B}^{\operatorname{sink}}(t)$ in eq. (2.1) is given by

$$
\mathcal{J}_{B B}^{\operatorname{sink}}(t)=\sum_{\vec{r}} \sum_{\vec{x}} B(\vec{x}+\vec{r}, t) B(\vec{x}, t)
$$

where a baryon operator $B$ is given in eq. (2.13). While plateau-like structures appear around $t / a \sim 15$ for both wall and smeared sources, the values disagree with each other. Similar inconsistencies are found on other volumes and the infinite volume extrapolation implies that the system is bound (unbound) for the smeared (wall) source. These discrepancies indicate some uncontrolled systematic errors. Indeed, such an early-time pseudoplateau can be shown to appear even with $10 \%$ contamination of the excited states as demonstrated by the mockup data [1].

Figure 1 (right) shows the sink operator dependence of $\Delta E_{\text {eff }}(t)$ for $\Xi \Xi\left({ }^{1} \mathrm{~S}_{0}\right)$ with the smeared source fixed, where the sink operator is generalized as

$$
\begin{aligned}
\mathcal{J}_{B B}^{\text {sink }}(t) & =\sum_{\vec{r}} g(\vec{r}) \sum_{\vec{x}} B(\vec{x}+\vec{r}, t) B(\vec{x}, t), \\
g(\vec{r}) & =1+\tilde{A} \exp (-\tilde{B} r)
\end{aligned}
$$

with four different parameter sets, $(\tilde{A}, \tilde{B})=(0.3,0.18),(-0.5,0.20),(-0.9,0.22)$ and $(0,0)$. The last one corresponds to the simple sink operator $(g(r)=1)$ in eq. (3.1). Although a plateau-like structure is observed for each sink operator, the values disagree with each other. This implies that the plateau-like behaviors at $t \simeq 1 \mathrm{fm}$ with the smeared source are not the plateau of the ground state but are pseudo-plateaux caused by contaminations of elastic scattering states other than the ground state. We note that such sink-operator dependence is not observed in the case of the wall source [1]. 

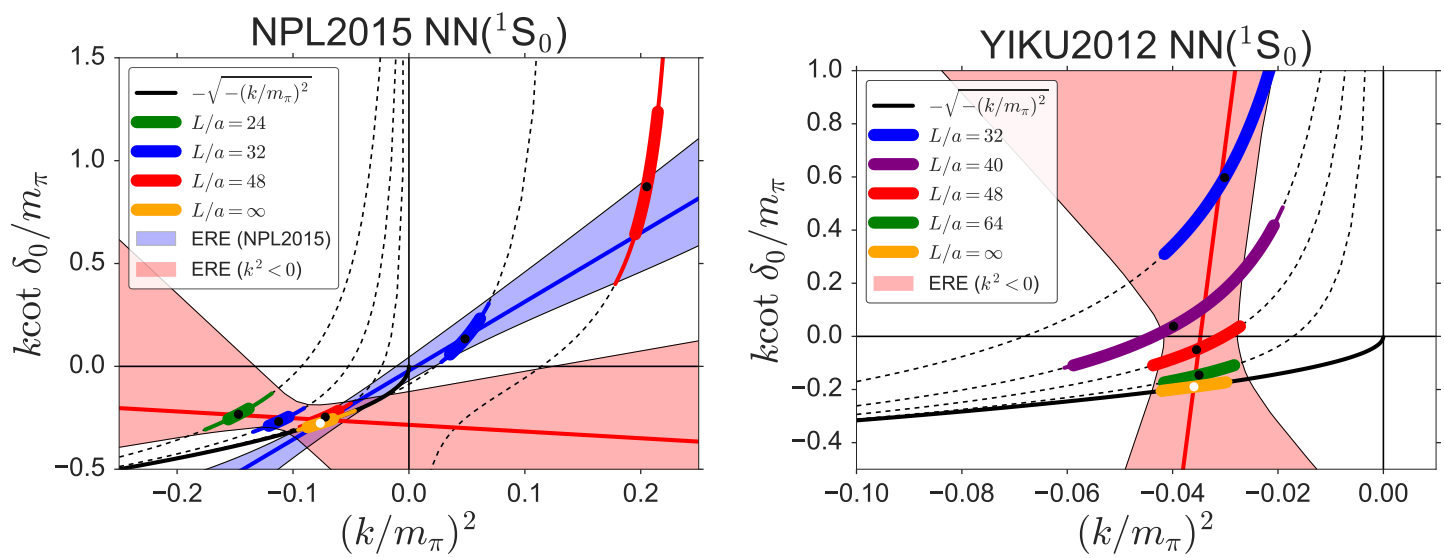

Figure 2. $k \cot \delta_{0}(k) / m_{\pi}$ as a function of $\left(k / m_{\pi}\right)^{2}$ for $N N\left({ }^{1} \mathrm{~S}_{0}\right)$ on each volume and the infinite volume in the direct method from ref. [30] (left) and ref. [28] (right). Black dashed lines correspond to Lüscher's formula for each volume, while the black solid line represents the bound-state condition, $-\sqrt{-\left(k / m_{\pi}\right)^{2}}$. The red line (with an error band) corresponds to the ERE obtained from the data at $k^{2}<0$ on finite volumes. In the left figure, the ERE fit to the data at $k^{2}>0$ on finite volumes together with only the infinite volume limit at $k^{2}<0$ is also shown by the blue line. Both figures from ref. [2].

\subsection{Normality check}

Since the information on operator dependence as shown in the previous subsection is not always available, we have introduced the "normality check" in ref. [2], based on Lüscher's finite volume formula together with the analytic properties of the $S$-matrix.

Some examples of the normality check are given in figure 2, where $k \cot \delta_{0}(k)$ is plotted as a function of $k^{2}$ for $N N\left({ }^{1} \mathrm{~S}_{0}\right)$. Red and blue lines in figure 2 represent fits to data by the effective range expansion (ERE) at the next-to-leading order (NLO) as

$$
k \cot \delta_{0}(k)=\frac{1}{a_{0}}+\frac{r_{0}}{2} k^{2},
$$

where $a_{0}$ and $r_{0}$ are the scattering length and the effective range, respectively. In figure 2 (left), inconsistency in ERE parameters is observed: the NLO ERE fit obtained from the data at $k^{2}<0$ on finite volumes (red line) disagrees with the fit to the data at $k^{2}>0$ on finite volumes together with the infinite volume limit at $k^{2}<0$ (blue line). For the latter fit (the blue line), the physical condition of the bound state pole is also violated. In figure 2 (right), the NLO ERE fit exhibits a singular behavior as the divergent effective range. See ref. [2] for more detailed discussions.

As in the case of operator dependence, the normality check in ref. [2] indicates that the plateau fitting at $t \simeq 1 \mathrm{fm}$ suffers from large uncontrolled systematic errors probably due to contaminations from the excited states.

\subsection{Source-operator dependence and derivative expansion of the potentials}

In ref. [20], we investigated source operator dependence of the potential as a tool to estimate the systematics associated with the derivative expansion of the HAL QCD potential, using two sources, wall and smeared sources. 

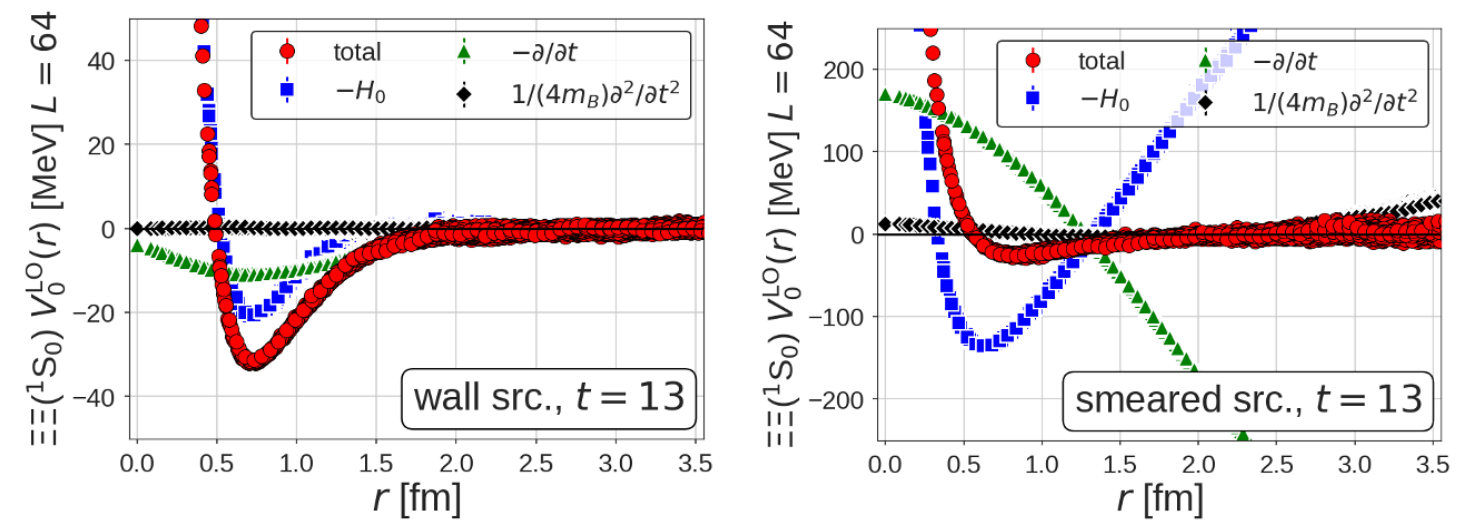

Figure 3. The potential at the leading order (LO) analysis $V_{0}^{\mathrm{LO}}(r)$ (red circles) from the wall source (left) and the smeared source (right) for $\Xi \Xi\left({ }^{1} \mathrm{~S}_{0}\right)$ at $t / a=13$ for $L=64$ [20]. The blue squares, green triangles and black diamonds denote 1st, 2nd and 3rd terms in eq. (2.11), respectively.

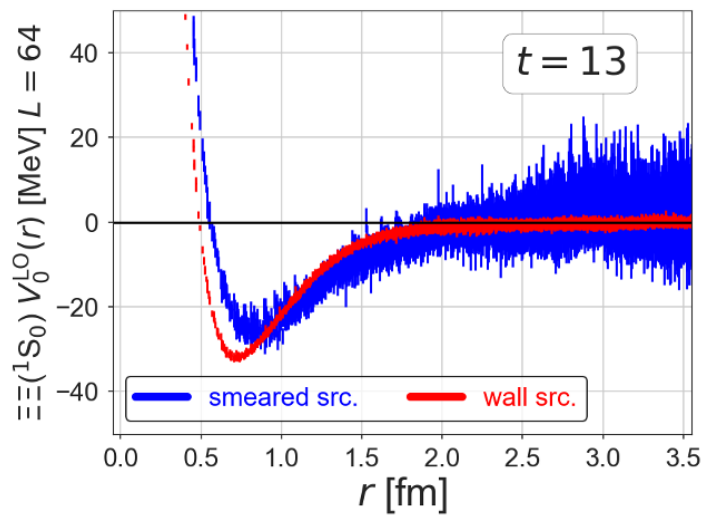

Figure 4. A comparison of the LO $\Xi \Xi\left({ }^{1} \mathrm{~S}_{0}\right)$ potential $V_{0}^{\mathrm{LO}}(r)$ between the wall source (red) and the smeared source (blue) at $t / a=13$ [20].

Figure 3 shows the LO $\Xi \Xi\left({ }^{1} \mathrm{~S}_{0}\right)$ potential and its breakup into 1st, 2nd, and 3rd terms in eq. (2.11) of the time dependent HAL QCD method from the wall source (left) and the smeared source (right). For the wall source, the 1st term dominates with moderate (negligible) contributions from the $2 \mathrm{nd}$ (3rd) term. As the 2 nd term is not constant as a function of $r$, there exist small but non-negligible contributions from the excited states. For the smeared source, on the other hand, all terms are important. The substantial $r$ dependence of the 2nd term (green triangles), which indicates large contributions from the excited states in the smeared source, is canceled by the 1st term (blue squares) and further corrected by the $3 \mathrm{rd}$ term (black diamonds). The total potentials (red circles) from two sources, however, show qualitatively similar behaviors, which illustrates that the time-dependent HAL QCD method works well for extracting the potential irrespective of the source types.

Figure 4 shows a comparison of the total potentials between two sources, $V_{0}^{\mathrm{LO} \text { (wall/smear) }}(r)$, at $t / a=13$. The potential approaches to zero within errors at larger $r$ for 

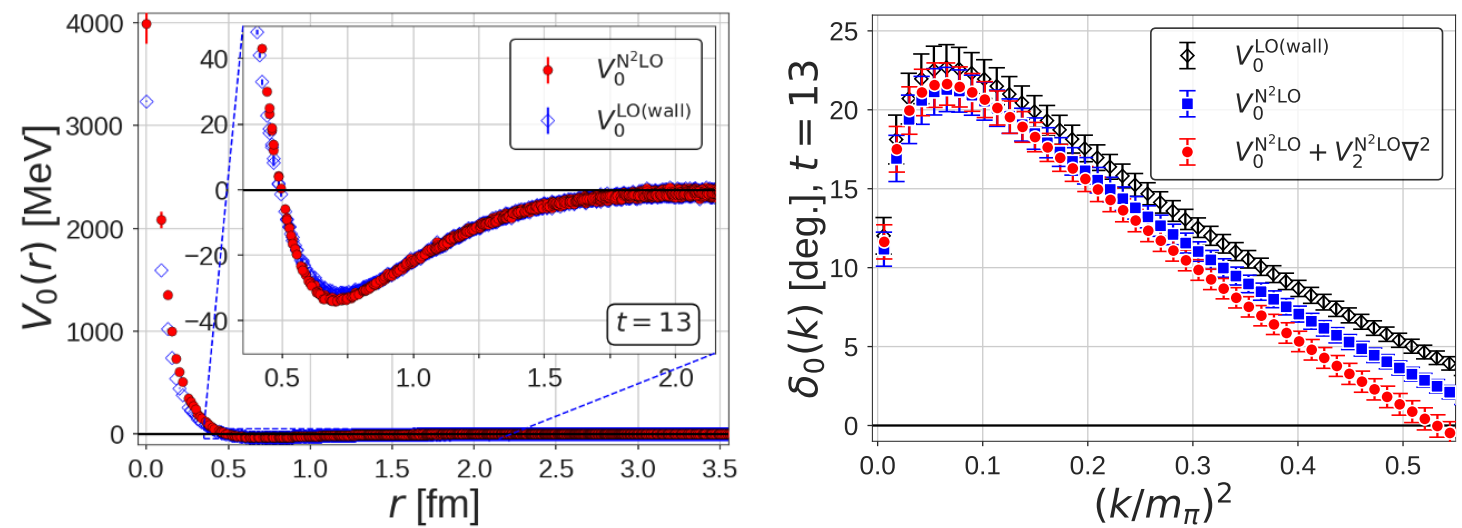

Figure 5. (Left) The LO $\Xi \Xi\left({ }^{1} \mathrm{~S}_{0}\right)$ potential at the $\mathrm{N}^{2} \mathrm{LO}$ analysis, $V_{0}^{\mathrm{N}^{2} \mathrm{LO}}(r)$ (red circles), together with the potential at the $\mathrm{LO}$ analysis for the wall source, $V_{0}^{\mathrm{LO}(\text { wall })}(r)$ (blue diamonds) at $t / a=13$ on $L=64$. (Right) The scattering phase shifts $\delta_{0}(k)$ from $V_{0}^{\mathrm{LO}(\text { wall })}$ (black diamonds), $V_{0}^{\mathrm{N}^{2} \mathrm{LO}}(r)$ (blue squares) and $V_{0}^{\mathrm{N}^{2} \mathrm{LO}}(r)+V_{2}^{\mathrm{N}^{2} \mathrm{LO}}(r) \nabla^{2}$ (red circles) at $t / a=13$. Both figures from ref. [20].

both sources, indicating that contributions from inelastic states are suppressed. While the potentials from two sources are very similar, there exists the non-zero difference between them. We find that this difference becomes smaller as $t$ increases, where the potential from the wall (smeared) source is independent of (dependent on) $t[20]$. This indicates that the effects of higher order terms in the derivative expansion exist in the data from the smeared source. Using the difference, $V_{0}^{\mathrm{N}^{2} \mathrm{LO}}(r)$ and $V_{2}^{\mathrm{N}^{2} \mathrm{LO}}(r)$ in eq. (2.12) can be determined. In figure 5 (left), $V_{0}^{\mathrm{N}^{2} \mathrm{LO}}(r)$ together with $V_{0}^{\mathrm{LO}(\text { wall) }}(r)$ on $L=64$ at $t / a=13$ are plotted. As we find that $V_{0}^{\mathrm{N}^{2} \mathrm{LO}}(r)$ agrees well with $V_{0}^{\mathrm{LO} \text { (wall) }}(r)$ except at short distances, we expect that $V_{0}^{\mathrm{LO}(\text { wall })}(r)$ works well to reproduce physical observables at low energies. Indeed, as shown in figure 5 (right), the $\mathrm{N}^{2} \mathrm{LO}$ correction to the S-wave scattering phase shift $\delta_{0}$ is small at low energies, showing not only that the derivative expansion converges well but also that the LO analysis for the wall source is sufficiently good at low energies.

\section{Anatomy: excited state contaminations in the effective energy shifts}

We now show our main results of this paper, where we analyze the behaviors of $\Xi \Xi\left({ }^{1} \mathrm{~S}_{0}\right)$ temporal correlation functions for both wall and smeared sources using the HAL QCD potential and demonstrate that contaminations of elastic excited states cause pseudo-plateau behaviors at early time slices.

The strategy of our analysis is as follows. Provided that the leading order HAL QCD potential from the wall source is found to be a reasonable approximation of the exact potential, we evaluate eigenfunctions of the Hamiltonian with this potential in the finite box whose eigenvalues are below the inelastic threshold. We then calculate overlap factors between these eigenmodes and the $\Xi \Xi\left({ }^{1} \mathrm{~S}_{0}\right)$ correlation functions, in terms of which we reconstruct pseudo-plateau behaviors of the energy shifts. We also show that the plateaux of the temporal correlation functions projected to the lowest or the 2nd lowest eigenfunction agree with their eigenvalues. This fact demonstrates that both HAL QCD potential 


\begin{tabular}{|c|ccc|}
\hline$\Delta E_{n}[\mathrm{MeV}]$ & $L=40$ & $L=48$ & $L=64$ \\
\hline$n=0$ & $-5.5(1.0)\left({ }_{-0.4}^{+1.8}\right)$ & $-2.8(0.4)\left({ }_{-0.1}^{+1.1}\right)$ & $-1.5(0.3)\left({ }_{-0.1}^{+0.4}\right)$ \\
$n=1$ & $77.2(0.8)\left({ }_{-4.7}^{+0.8}\right)$ & $52.0(0.4)\left(\left(_{0.0}^{+3.6}\right)\right.$ & $28.4(0.3)\left({ }_{-0.1}^{+0.4}\right)$ \\
$n=2$ & $161.5(1.0)\left({ }_{-6.8}^{+0.2}\right)$ & $110.0(0.5)\left({ }_{0.0}^{+3.2}\right)$ & $60.4(0.4)\left({ }_{-0.1}^{+0.3}\right)$ \\
$n=3$ & $236.5(1.1)\left({ }_{-0.7}^{+1.0}\right)$ & $164.9(0.6)\left({ }_{-0.5}^{+2.1}\right)$ & $93.2(0.4)\left(\left(_{0.0}^{+0.7}\right)\right.$ \\
$n=4$ & - & $216.3(0.4)\left({ }_{-0.4}^{+1.0}\right)$ & $124.1(0.3)\left({ }_{-0.1}^{+0.2}\right)$ \\
$n=5$ & - & - & $155.8(0.3)\left({ }_{-0.0}^{+0.6}\right)$ \\
$n=6$ & - & - & $186.5(0.3)\left(\begin{array}{l}+0.8 \\
0.0\end{array}\right)$ \\
\hline
\end{tabular}

Table 2. Eigenvalues of the $n$-th eigenfunction below the inelastic threshold in the $A_{1}^{+}$representation of the HAL QCD Hamiltonian $H^{\mathrm{LO}}$ in each volume. Eigenvalues are given in terms of the energy shifts from the threshold, $\Delta E_{n} \equiv W_{n}-2 m_{\Xi}$. Central values and statistical errors are evaluated at $t / a=13$, while the systematic errors are estimated by using the results at $t / a=14,15,16$.

method and accurate extraction of energy shifts from the temporal correlation function give consistent results.

\subsection{Eigenvalues and eigenfunctions in the finite box}

We first evaluate eigenvalues and eigenfunctions of the leading-order HAL QCD Hamiltonian in a finite box given by

$$
H^{\mathrm{LO}}=H_{0}+U, \quad U \equiv V_{0}^{\mathrm{LO}(\text { wall })},
$$

where we take $V_{0}^{\mathrm{LO} \text { (wall) }}$ on each volume for $U$, since $V_{0}^{\mathrm{LO}(\text { wall) }}$ is a reasonable approximation of the exact potential $U .^{5}$ Note that the volume dependence of $V_{0}^{\mathrm{LO} \text { (wall) }}$ is negligible, thanks to the short-ranged nature of the interaction.

In a finite lattice box, eigenvalues and eigenfunctions of the Hermitian matrix $H^{\mathrm{LO}}$ can be easily obtained. The $n$-th eigenvalue of $H^{\mathrm{LO}}$ in the $A_{1}^{+}$representation below the inelastic threshold ${ }^{6}$ is tabulated in table 2 , where we show the energy shift compared to the threshold, $\Delta E_{n} \equiv W_{n}-2 m_{\Xi}$. The number of excited states below the inelastic threshold is 3,4 and 6 on $L=40,48$, and 64, respectively. For larger volume, the energy gap becomes smaller and the number of elastic excited states increases.

Figure 6 shows the eigenfunctions $\Psi_{n}(\vec{r})$ on $L=48$, with $\sum_{\vec{r}}\left|\Psi_{n}(\vec{r})\right|^{2}=1$ and $\Psi_{n}(\overrightarrow{0})>0 .^{7}$ Up to a normalization, $\Psi_{n}(\vec{r})$ corresponds to the NBS wave function $\psi^{W=W_{n}}(\vec{r})$ in a finite volume. The lowest state $\Psi_{0}(\vec{r})$ has the similar shape to the $R$-correlator for

\footnotetext{
${ }^{5}$ In appendix A, we employ the potential at the $\mathrm{N}^{2} \mathrm{LO}$ analysis instead, and find that the results are consistent with the LO results.

${ }^{6}$ For the $\Xi \Xi$ system in the ${ }^{1} S_{0}$ channel at $m_{\pi}=0.51 \mathrm{GeV}$, the lowest inelastic threshold is either $\Xi^{*} \Xi$ or $\Sigma \Omega$ in ${ }^{5} \mathrm{D}_{0}$ channel, which corresponds to $W_{\mathrm{th}}-2 m_{\Xi} \simeq 0.25-0.30 \mathrm{GeV}$ on $L=64-40$, using $m_{\Sigma}=1.40 \mathrm{GeV}, m_{\Xi^{*}}=1.68 \mathrm{GeV}$ and $m_{\Omega}=1.74 \mathrm{GeV}$.

${ }^{7} \Psi_{n}(\vec{r})$ is a multi-valued function of $r$ due to the effect of the (periodic) finite box.
} 

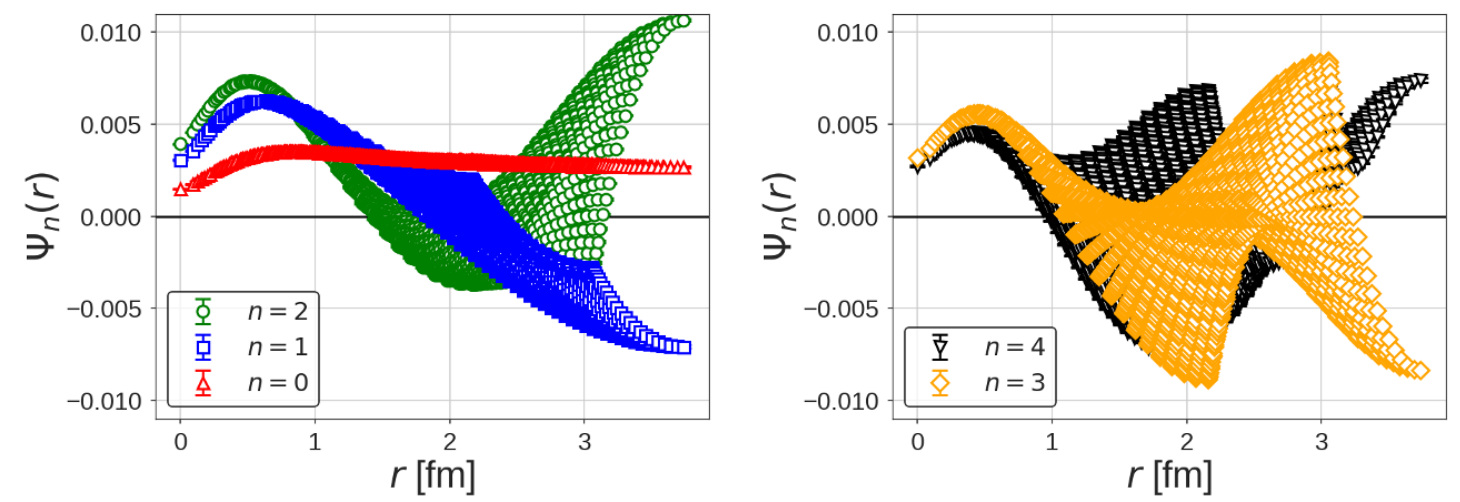

Figure 6. Eigenfunctions of elastic states in the $A_{1}^{+}$representation of the HAL QCD Hamiltonian $H^{\mathrm{LO}}$ below the inelastic threshold $\Psi_{n}(\vec{r})$ on $L=48$ at $t / a=13$ for $n=0,1,2$ (left) and $n=$ 3,4 (right), where red up-pointing triangles, blue squares, green circles, orange diamonds and black down-pointing triangles correspond to $n=0,1,2,3$ and 4 , respectively. The eigenfunction is normalized as $\sum_{\vec{r}}\left|\Psi_{n}(\vec{r})\right|^{2}=1$ and $\Psi_{n}(\overrightarrow{0})>0$. Errors are statistical only.

the wall source, which shows a weak peak structure around $r \lesssim 1 \mathrm{fm}$ and becomes flat at large distances without any nodes, while the eigenfunctions for the excited states have nodes, whose number increases as the eigenvalue becomes larger. The short distance structures for $\Psi_{n>0}(\vec{r})$, which has a steeper peak around $r<1 \mathrm{fm}$ than that of $\Psi_{0}(\vec{r})$ resemble the $R$-correlator for the smeared source. Eigenfunctions on other volumes are collected in appendix B.

\subsection{Decomposition of the $R$-correlator via eigenfunctions}

Since the $R$-correlator is dominated by elastic states at moderately large $t$, we can expand it in terms of eigenfunctions of $H^{\mathrm{LO}}$ as

$$
R^{\text {wall } / \text { smear }}(\vec{r}, t)=\sum_{n} a_{n}^{\text {wall/smear }} \Psi_{n}(\vec{r}) e^{-\Delta E_{n} t},
$$

where the overlap coefficient $a_{n}$ characterizes the magnitude of the contribution from the corresponding eigenfunction. Using the orthogonality of $\Psi_{n}(\vec{r}), a_{n}$ can be determined by

$$
a_{n}^{\text {wall/smear }}=\sum_{\vec{r}} \Psi_{n}^{\dagger}(\vec{r}) R^{\text {wall/smear }}(\vec{r}, t) e^{\Delta E_{n} t} .
$$

The magnitude of the corresponding excited state contamination in the $R$-correlator is represented by the ratio $a_{n} / a_{0}$. In figure 7 , we plot $a_{n} / a_{0}$ obtained at $t / a=13$ as a function of $\Delta E_{n}$ for the wall source (left) and the smeared source (right). Calculations at $t / a=14,15,16$ confirm that the results are almost independent of $t$ within statistical errors, indicating that the decomposition is reliable. ${ }^{8}$ In the case of the wall source, $R^{\text {wall }}(r, t)$ has a large overlap with the ground state, and $\left|a_{n>0} / a_{0}\right|$ is smaller than 0.1 . In the case of

\footnotetext{
${ }^{8}$ In appendix C, we check how well the decomposition in eq. (4.2) approximates the original $R$-correlator. It is found that the magnitude of the residual relative to the original $R$-correlator at $t / a=13$ is as small as $\mathcal{O}\left(10^{-5}-10^{-6}\right)$ for the wall source and $0.4 \%, 2 \%, 5 \%$ for the smeared source on $L=40,48,64$, respectively.
} 

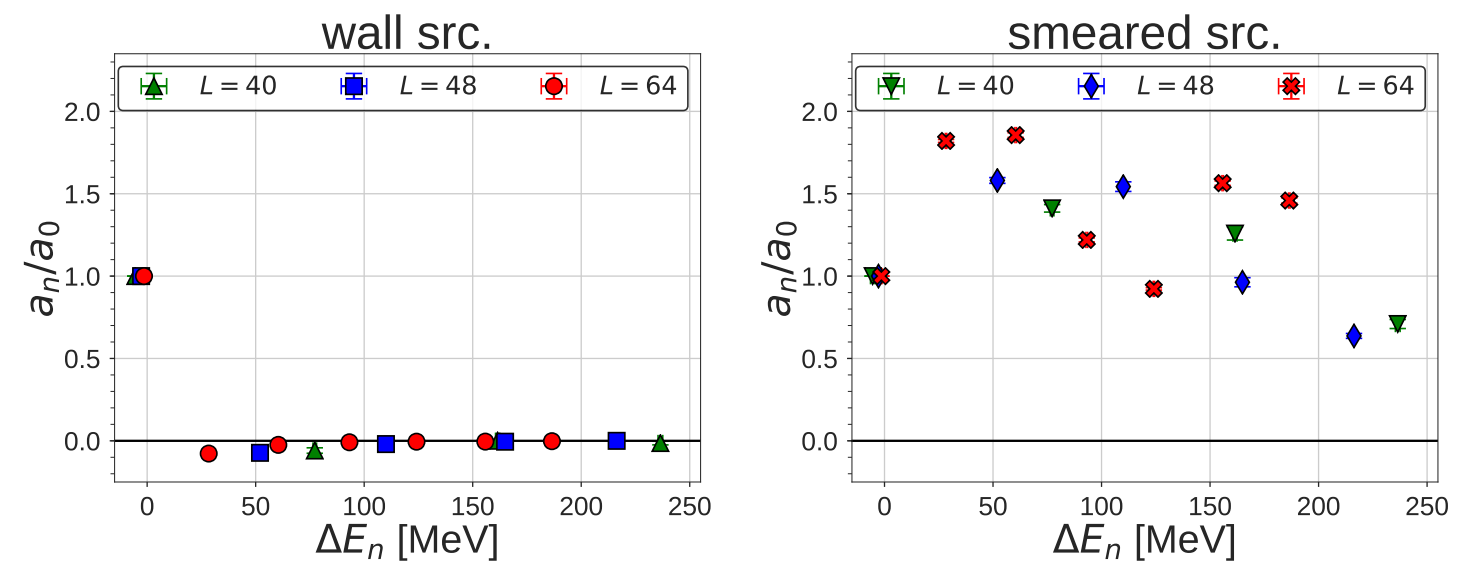

Figure 7. The ratio of the overlap coefficients $a_{n} / a_{0}$ in the $R$-correlator obtained at $t / a=13$ for the wall source (left) and the smeared source (right) on three volumes.
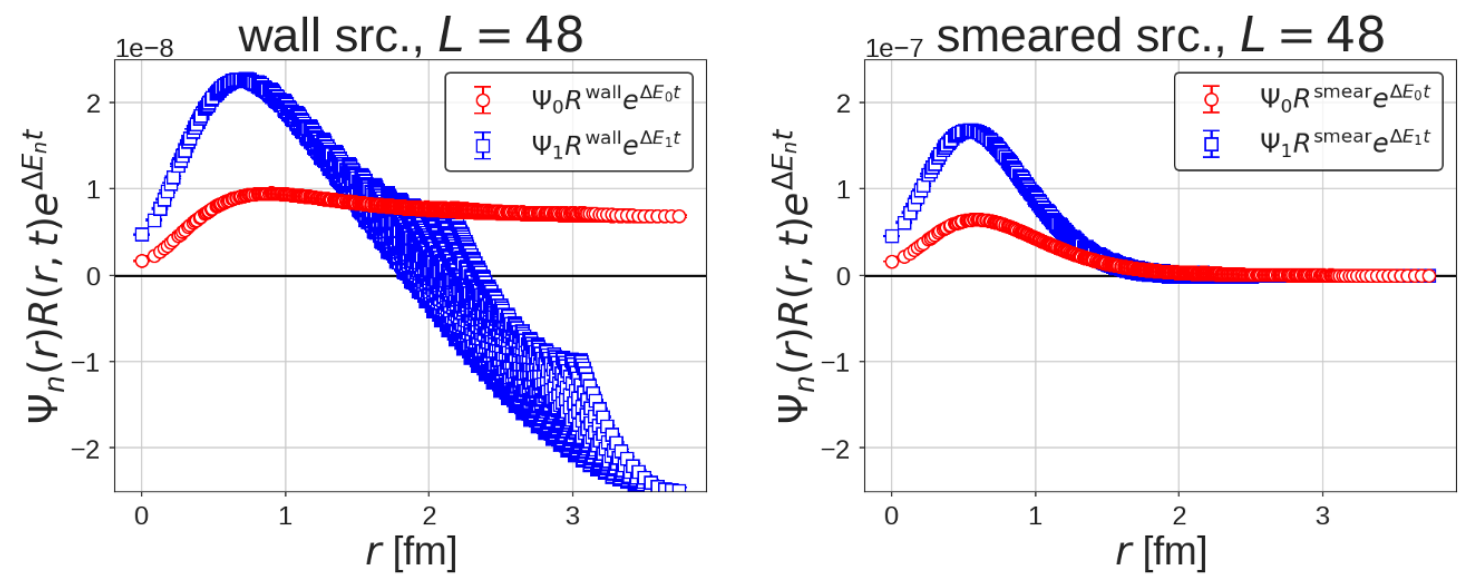

Figure 8. The overlap between the $R$-correlator and the eigenfunction, $\Psi_{n}^{\dagger}(\vec{r}) R(\vec{r}, t) e^{\Delta E_{n} t}$, as a function of $r$ at $t / a=13$ on $L=48$ for the ground state $(n=0$, red circles) and the first excited state ( $n=1$, blue squares) in the case of the wall source (left) and the smeared source (right).

the smeared source, on the other hand, $\left|a_{n} / a_{0}\right| \sim \mathcal{O}(1)$ and thus all elastic excited states significantly contribute to the $R$-correlator.

This difference of the magnitude in $a_{n} / a_{0}$ between two sources can be understood from the overlap between the $R$-correlator and the eigenfunctions. Figure 8 shows the spatial profile of the overlap, $\Psi_{n}^{\dagger}(\vec{r}) R(\vec{r}, t) e^{\Delta E_{n} t}$, for the wall source (left) and the smeared source (right) on $L=48$, whose spatial summation corresponds to $a_{n}$. The contribution from the first excited state (blue squares) is highly suppressed for the wall source, thanks to the cancellation between positive values at short distances and negative values at long distances of $\Psi_{n=1}^{\dagger}(\vec{r}) R^{\text {wall }}(\vec{r}, t) e^{\Delta E_{n=1} t}$. For the smeared source, on the contrary, the contamination from the 1st excited state remains non-negligible, due to the absence of the negative parts in $\Psi_{n=1}^{\dagger}(\vec{r}) R^{\text {smear }}(\vec{r}, t) e^{\Delta E_{n=1} t}$. 
In the literature, the smeared source is often employed in the direct method, in order to suppress contributions from (inelastic) excited states in a "single-baryon" correlation function. The same smeared source, however, does not necessarily reduce the contaminations from the elastic excited states in the two-baryon correlation function, as is explicitly shown in figure 7 . Indeed, one of the most relevant parameters which control the magnitudes of elastic state contributions is the relative distance $\vec{r}$ between two baryons at the source, which appears as

$$
\frac{1}{L^{3}} \sum_{\vec{x}} B(\vec{x}) B(\vec{x}+\vec{r})=\sum_{\vec{p}} \tilde{B}(\vec{p}) \tilde{B}(-\vec{p}) e^{i \vec{p} \cdot \vec{r}}, \quad \tilde{B}(\vec{p}) \equiv \frac{1}{L^{3}} \sum_{\vec{x}} B(\vec{x}) e^{-i \vec{p} \cdot \vec{x}}
$$

in the center of mass system. Almost all literature of the direct method have employed the smeared source essentially corresponding to $|\vec{r}|=0$, which implies that elastic states for all $\vec{p}$ are equally generated at the source. Thus the choice $|\vec{r}|=0$ (or $|\vec{r}| \ll 1$ ) is one of the possible reasons for large contaminations from elastic excited states in the case of the smeared source. As long as $|\vec{r}|$ is non-zero and large, however, modes with non-zero $\vec{p}$ may be suppressed due to the oscillating factor $e^{i \vec{p} \cdot \vec{r}} \cdot{ }^{9}$ The study in which the momentum projection is performed for each baryon in the source operator is recently reported in ref. [16].

The temporal correlation function $R(t)$ is reconstructed in terms of eigenfunctions as

$$
R^{\text {wall } / \text { smear }}(t)=\sum_{\vec{r}} R^{\text {wall } / \text { smear }}(\vec{r}, t)=\sum_{\vec{r}} \sum_{n} a_{n}^{\text {wall } / \text { smear }} \Psi_{n}(\vec{r}) e^{-\Delta E_{n} t}=\sum_{n} b_{n}^{\text {wall } / \text { smear }} e^{-\Delta E_{n} t},
$$

where $b_{n} \equiv a_{n} \sum_{\vec{r}} \Psi_{n}(\vec{r})$, whose ratio $b_{n} / b_{0}$ gives the magnitude of the contamination to $R(t)$ from the $n$-th elastic excited state.

Figure 9 shows $\left|b_{n} / b_{0}\right|$ obtained at $t / a=13$ as a function of $\Delta E_{n}$ on three volumes for the wall source (left) and the smeared source (right). Solid (open) symbols correspond to positive (negative) values for $b_{n} / b_{0}$. For the wall source, the contamination from the first excited state is found to be smaller than $1 \%$, and $\left|b_{n} / b_{0}\right|$ is further suppressed exponentially for higher excited states. In the case of the smeared source, the contamination from the first excited state is as large as $\sim 10 \%$ with a negative sign and the contamination remains to be $\sim 1 \%$ even for the higher excited state with $\Delta E_{n} \sim 100 \mathrm{MeV}$.

\subsection{Reconstruction of the effective energy shift}

Let us now examine the energy shifts obtained from the reconstructed $R$-correlators;

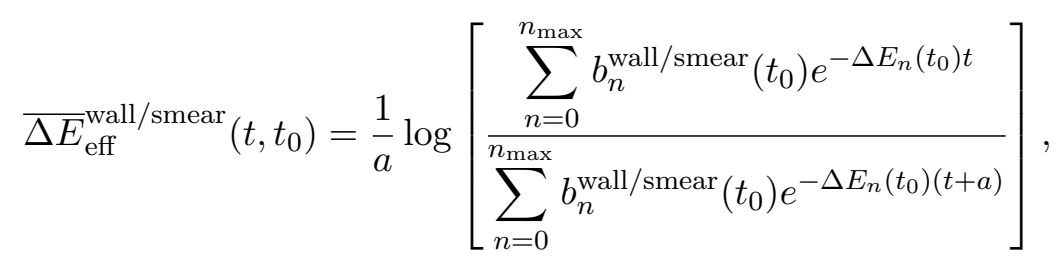

\footnotetext{
${ }^{9}$ Ref. [5] reported the discrepancy in the effective energies between the zero displaced $(|\vec{r}|=0)$ and the non-zero displaced $(|\vec{r}| \neq 0)$ source operators, which can be naturally understood from this viewpoint, rather than the existence of two bound states claimed in ref. [5].
} 

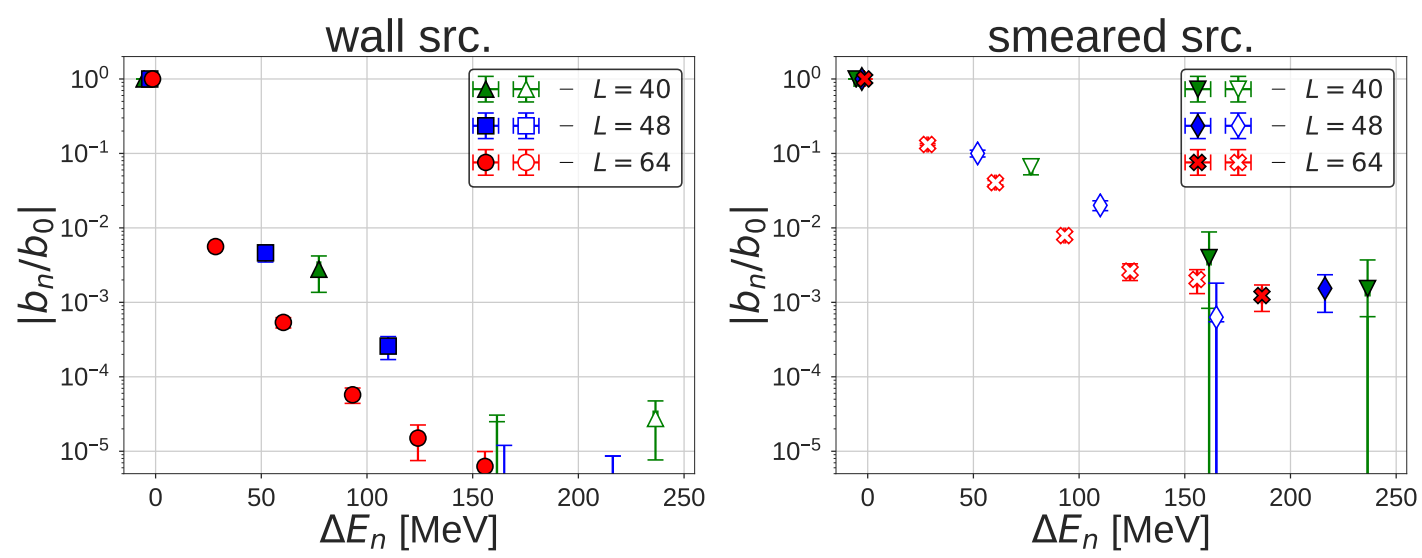

Figure 9. The ratio of the overlap coefficients in the temporal correlation function $\left|b_{n} / b_{0}\right|$ obtained at $t / a=13$ for the wall source (left) and the smeared source (right) on various volumes. Solid (open) symbols correspond to positive (negative) values of $b_{n} / b_{0}$.
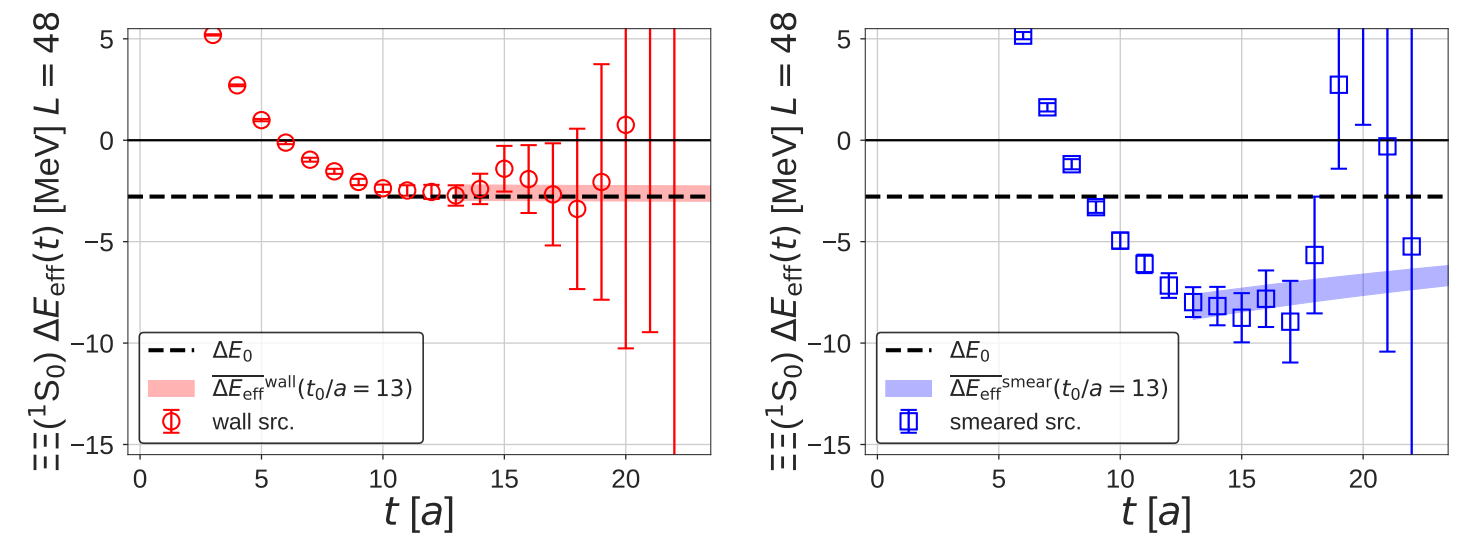

Figure 10. The reconstructed effective energy shifts $\overline{\Delta E}_{\text {eff }}\left(t, t_{0}=13 a\right)$ with statistical errors are plotted as a function of $t$ (colored bands), while the direct measurement of the effective energy shifts from $R$-correlators are plotted by red circles or blue squares. The black dashed lines correspond to the energy shift $\Delta E_{0}\left(t_{0}=13 a\right)$ for the ground state of the HAL QCD Hamiltonian $H^{\mathrm{LO}}$ in the finite volume. The results on $L=48$ for the wall source (left) and the smeared source (right).

where we take $n_{\max }=3,4,6$ for $L=40,48,64$, respectively, corresponding to the number of elastic excited states below the inelastic threshold, and $b_{n}\left(t_{0}\right)$ and $\Delta E_{n}\left(t_{0}\right)$ are extracted at fixed $t_{0}$.

In figure 10, we show the reconstructed effective energy shifts $\overline{\Delta E}_{\text {eff }}\left(t, t_{0}=13 a\right)$, together with numerical data of the effective energy shifts $\Delta E_{\text {eff }}(t)$ from the $R$-correlators, for the wall source (left) and the smeared source (right) on $L=48$. The bands correspond to $\overline{\Delta E}_{\text {eff }}\left(t, t_{0}=13 a\right)$ with statistical errors coming from those of $b_{n}$ and $\Delta E_{n}$ at $t_{0} / a=13$, while red circles or blue squares correspond to $\Delta E_{\text {eff }}(t)$ obtained directly from the $R$ correlator in section 3.1. Here we do not consider $\overline{\Delta E}_{\text {eff }}\left(t, t_{0}=13 a\right)$ for $t / a<13$, where inelastic contributions are expected to be larger. Shown together by the black dashed line represents the energy shift $\Delta E_{0}\left(t_{0}=13 a\right)$ for the ground state of the HAL QCD Hamiltonian $H^{\mathrm{LO}}$ on $L=48$. 

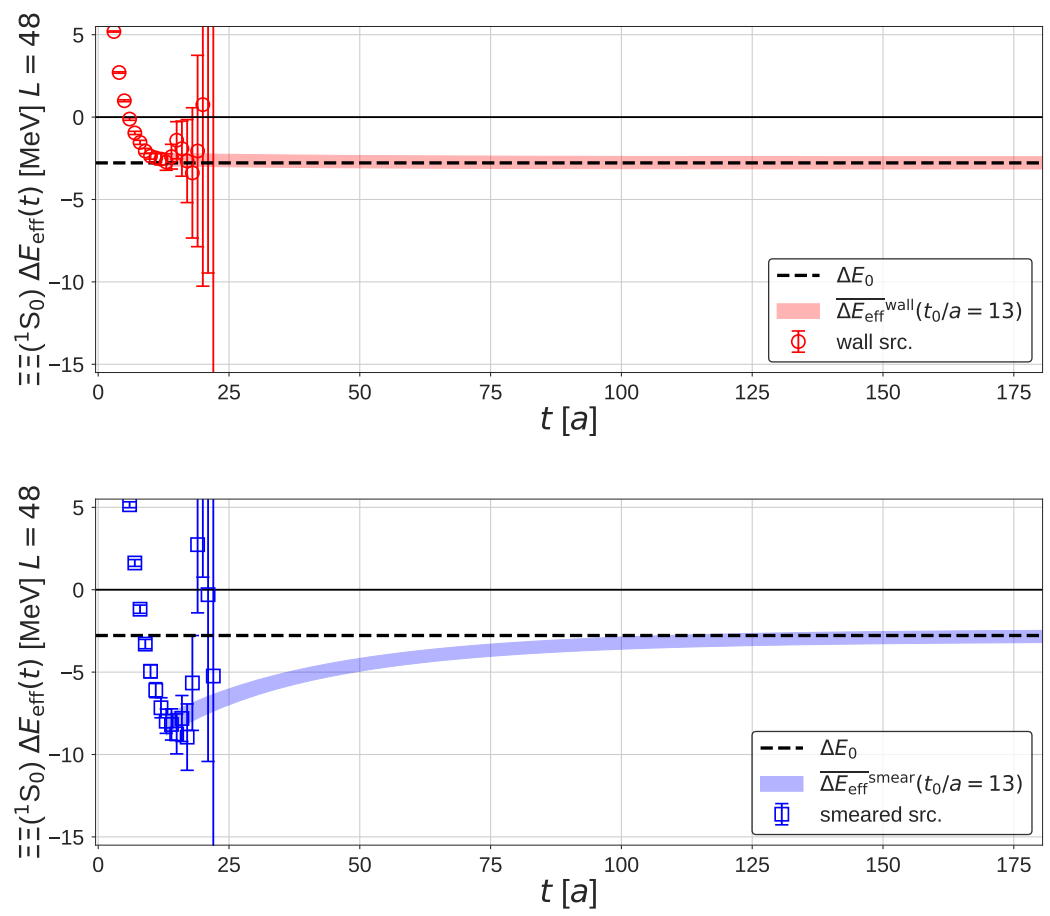

Figure 11. The same as figure 10 but for the wider range of the Euclidean time $t$.

We find that the results of the direct method, most notably the plateau-like structures around $t / a=15$, are well reproduced by $\overline{\Delta E}_{\text {eff }}\left(t, t_{0}\right)$ for both wall and smeared sources, indicating that the behaviors of $\Delta E_{\text {eff }}(t)$ at this time interval in the direct method are explained by the contributions from the several low-lying states. These plateau-like structures, however, do not necessarily correspond to the plateau of the ground state. Indeed, in the case of the smeared source, there is a clear discrepancy between the value of the plateau-like structure and the eigenvalue $\Delta E_{0}$ of the ground state. This is a consequence of large excited state contaminations in the correlation function for the smeared source. In the case of the wall source, on the other hand, since the overlap with the ground state is large, the value of the plateau-like structure is consistent with the value $\Delta E_{0}$.

The fate of the plateau-like structures is more clearly seen in figure 11, where we plot the behaviors at asymptotically large $t$ of the reconstructed effective energy shifts $\overline{\Delta E}_{\text {eff }}\left(t, t_{0}=13 a\right)$ for the wall source (red band) and the smeared source (blue band). While the plateau-like structure at $t / a \sim 15$ for the wall source is almost unchanged at larger $t$, the value of $\overline{\Delta E}_{\text {eff }}\left(t, t_{0}=13 a\right)$ in the case of the smeared source gradually changes as $t$ increases until it reaches to the value of the ground state, $\Delta E_{0}$, at $t / a \sim 100$.

In appendix D.1, we perform the same analysis on other volumes and observe essentially the same behaviors as in the case of $L=48$ : for the wall source, the value of the plateaulike structure at $t / a \sim 15$ remains almost unchanged at larger $t$ and is consistent with $\Delta E_{0}$. For the smeared source, the value of the plateau-like structure at $t / a \sim 15$ is inconsistent with $\Delta E_{0}$. The deviation is found to be larger on a larger volume, due to 
severer contaminations from the excited states on larger volumes (see section 4.2). ${ }^{10}$ The value of $\overline{\Delta E}_{\text {eff }}\left(t, t_{0}=13 a\right)$ for the smeared source gradually changes at $t$ increases and the ground state saturation is realized at $t / a \gtrsim 50,100$ and 150 or $t \gtrsim 5,10$ and $15 \mathrm{fm}$ on $L=$ 40, 48 and 64, respectively. These time scales for the ground state saturation are actually not surprising but rather natural, considering the fact that the lowest excitation energy is as small as $\delta E \equiv \Delta E_{1}-\Delta E_{0} \simeq 84,55$ and $30 \mathrm{MeV}$ on $L=40,48$ and 64 , respectively.

These results clearly reveal that the plateau-like structures at $t / a \sim 15$ for the smeared source are pseudo-plateaux caused by contaminations of the elastic excited states. ${ }^{11}$ While the effective energy shifts from the wall source happen to be saturated by the ground state even at $t / a \sim 15$, it is generally difficult to confirm that a plateau-like structure corresponds to the correct energy shift of the ground state without the help of other inputs, such as the HAL QCD potential analysis in the present case. Since the calculation of the energy shift from the $R$-correlator at $t \sim(\delta E)^{-1}$ is impractical due to the exponentially growing noises, one cannot obtain the correct spectra from the plateau identification in the direct method unless sophisticated variational techniques [32] are employed. ${ }^{12}$

\subsection{Projection with improved sink operator}

Once the finite-volume eigenmodes of $H^{\mathrm{LO}}$ with the HAL QCD potential are known, an improved two-baryon sink operator for a designated eigenstate can be constructed as

$$
\mathcal{J}_{B B}^{\operatorname{sink}}(t)=\sum_{\vec{r}} \Psi_{n}^{\dagger}(\vec{r}) \sum_{\vec{x}} B(\vec{x}+\vec{r}, t) B(\vec{x}, t)
$$

which is expected to have a large overlap to the $n$-th elastic state. ${ }^{13}$ This is equivalent to considering the generalized temporal correlation function with the choice of $g(\vec{r})=\Psi_{n}^{\dagger}(\vec{r})$ in eq. (3.2),

$$
R^{(n)}(t) \equiv \sum_{\vec{r}} \Psi_{n}^{\dagger}(\vec{r}) R(\vec{r}, t)
$$

from which we define the effective energy shift for the $n$-th eigenfunction as

$$
\Delta E_{\mathrm{eff}}^{(n)}(t)=\frac{1}{a} \log \frac{R^{(n)}(t)}{R^{(n)}(t+a)}
$$

\footnotetext{
${ }^{10}$ Values of pseudo-plateaux do not strongly depend on volumes, while the correct values $\Delta E_{0}$ do. This is a counter example against the argument in refs. $[4,31]$ in which it is claimed that the volume-independence of the plateaux guarantees their correctness.

${ }^{11}$ In appendix D.2, we show that the dominant contamination comes from the first excited state.

${ }^{12}$ Application of the variational method to two-baryon systems has started lately but mostly with respect to the flavor space [16]. It will be interesting to perform the variational method with respect to relative coordinate space for two baryons in addition. In the lattice study of meson-meson scatterings [33], the danger of the excited state contaminations in the plateau fitting has been already recognized and the use of the variational method is known to be mandatory.

${ }^{13}$ Use of such an improved operator as a "source" requires additional calculations and thus is left for future study.
} 

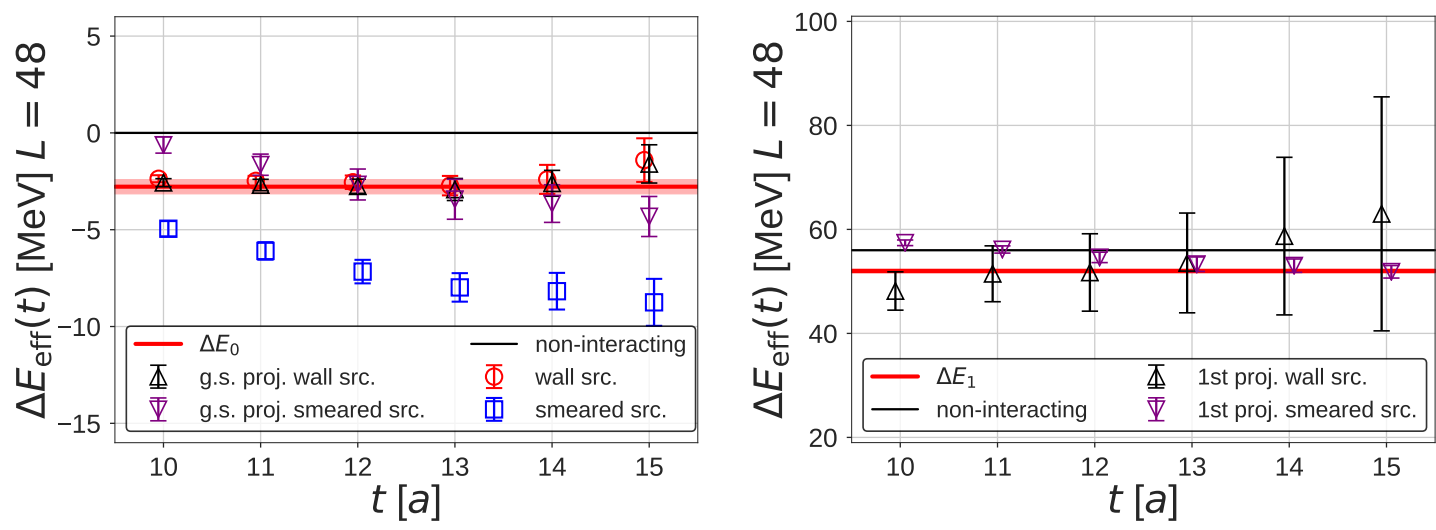

Figure 12. The effective energy shift $\Delta E_{\text {eff }}^{(n)}(t)$ on $L=48$ from the wall source (black up triangles) and the smeared source (purple down triangles) for the ground state (left) and the first excited state (right). Red bands represent the energy shifts with statistical errors obtained from the HAL QCD Hamiltonian $H^{\mathrm{LO}}$, while black lines represent those for a non-interacting system. In the left figure, the effective energy shifts in the direct method for the wall source (red circles) and the smeared source (blue squares) are also shown.

Figure 12 shows the effective energy shift $\Delta E_{\text {eff }}^{(n)}(t)$ using the wall source (black uptriangles) and the smeared sources (purple down-triangles) on $L=48$ for the ground state (left) and the first excited state (right). Shown together are the energy shift $\left(\Delta E_{0}\right.$ or $\Delta E_{1}$ ) with statistical errors (red bands), obtained from $H^{\mathrm{LO}}$ with the HAL QCD potential $V_{0}^{\mathrm{LO} \text { (wall) }}(r)$ at $t / a=13$, as well as that for a non-interacting system (black lines). In the case of the ground state (figure 12 (left)), the effective energy shifts from the direct method for the wall source (red circles) and the smeared source (blue squares) are also plotted for comparisons.

First of all, after the sink projections, the results with the wall source and those with the smeared source agree well around $t / a \sim 13$ not only for the ground state but also for the first excited state. This is in sharp contrast with the fact that the results in the direct method without projections disagree between two sources for the ground state. Although a small overlap with the first excited state causes relatively large statistical errors in the case of the wall source, an agreement between two sources for the first excited states is rather striking, and serves as a non-trivial check for the reliability of the effective energy shifts with the sink projection. Moreover, results after the sink projections also agree with those from the HAL QCD Hamiltonian. Although the sink projection utilizes the information of the HAL QCD potential through eigenfunctions, agreements in effective energy shifts within statistical errors for both ground and first excited states provide a non-trivial consistency check between the HAL QCD method and the direct method with proper projection. In other words, results in figure 12 establish that (i) the HAL QCD potential correctly describes the energy shifts of two baryons in the finite volume for both ground and excited states, and that (ii) these energy shifts can also be extracted in the direct method if and only if interpolating operators are highly improved. Since the origins of systematic uncertainties are generally quite different between the two methods, such a "projection check" would be useful in future lattice QCD studies for two-baryon systems. 
In recent years, it has been argued that seemingly inconsistent results for the $N N$ systems at heavy pion masses between Lüscher's finite volume method and the HAL QCD method may indicate some theoretical deficits in one of the two methods. It is now clear from our analysis that Lüscher's method and the HAL QCD method agree quantitatively with each other, as it should be so theoretically.

\section{Summary}

In our previous works $[1,2]$, it has been shown that the plateau fitting of the eigenenergies at early Euclidean times $t$, employed in the direct method, is generally unreliable for multibaryon systems, due to the appearance of pseudo-plateaux caused by contaminations of the excited states with small gap corresponding to the elastic scattering states on the finite volume. In this paper, we quantified the degree of contaminations from such excited states by decomposing the two-baryon correlation functions in terms of the finite-volume eigenmodes of the HAL QCD Hamiltonian.

By taking $\Xi \Xi\left({ }^{1} \mathrm{~S}_{0}\right)$ system at $m_{\pi}=0.51 \mathrm{GeV}$ in $(2+1)$-flavor lattice QCD with the wall and smeared quark sources for $L a=3.6,4.3,5.8 \mathrm{fm}$, we showed that the excited state contaminations are suppressed for the wall source, while those for the smeared source are substantial and become severer on a larger spatial extent. For the smeared source, the plateau-like structures at $t=1 \sim 2 \mathrm{fm}$ are shown to be pseudo-plateaux and the plateau with the ground state saturation is realized only at $t>5 \sim 15 \mathrm{fm}$ corresponding to the inverse of the lowest excitation energy. We also demonstrated that one can optimize the two-baryon operator utilizing the finite-volume eigenmode of the HAL QCD Hamiltonian. The effective energies from the temporal correlation functions with the optimized operators are found to be consistent with the finite volume spectra obtained from the HAL QCD Hamiltonian. This result establishes not only that the correct finite-volume spectra can be accessed by employing highly optimized operators even in the direct method but also that the HAL QCD method and the direct method agree in the finite volume spectra for the two baryon systems. Thus the long-standing issue on the consistency between Lüscher's finite volume method and the HAL QCD method is positively resolved at least for the particular system considered here. The next step is to carry out comprehensive studies of baryon-baryon interactions around the physical quark masses in the HAL QCD method, which are partly underway (see, e.g. [34-36]). Those will reveal not only the nature of exotic dibaryons but also the equation of state of dense baryonic matter. 


\section{Acknowledgments}

We thank the authors of ref. [28] and ILDG/JLDG [37-39] for providing the gauge configurations. Lattice QCD codes of CPS [40], Bridge++ [41] and the modified version thereof by Dr. H. Matsufuru, cuLGT [42] and domain-decomposed quark solver [43, 44] are used in this study. The numerical calculations have been performed on BlueGene/Q and SR16000 at KEK, HA-PACS at University of Tsukuba, FX10 at the University of Tokyo and K computer at RIKEN R-CCS (hp150085, hp160093). This work is supported in part by the Japanese Grant-in-Aid for Scientific Research (No. JP24740146, JP25287046, JP15K17667, JP16K05340, JP16H03978, JP18H05236, JP18H05407), by MEXT Strategic Program for Innovative Research (SPIRE) Field 5, by a priority issue (Elucidation of the fundamental laws and evolution of the universe) to be tackled by using Post K Computer, and by Joint Institute for Computational Fundamental Science (JICFuS).

\section{A The finite volume spectra from the $\mathrm{N}^{2} \mathrm{LO}$ potential}

In the main text (section 4), we study the finite volume spectra to be used for the spectral decomposition of the $R$-correlator using the LO potential. In this appendix, we employ the potential at the $\mathrm{N}^{2} \mathrm{LO}$ analysis (eq. (2.12)) and examine a stability of the finite volume spectra against the order of the derivative expansion. The HAL QCD Hamiltonian with the $\mathrm{N}^{2} \mathrm{LO}$ potential is given by

$$
H^{\mathrm{N}^{2} \mathrm{LO}}=H_{0}+V_{0}^{\mathrm{N}^{2} \mathrm{LO}}(r)+V_{2}^{\mathrm{N}^{2} \mathrm{LO}}(r) \nabla^{2} .
$$

Note that, while $H$ is non-Hermitian, its eigenenergies are real since the eigen equation can be rewritten as the definite generalized Hermitian eigenvalue problem [20]. The eigenenergies at $L=64$ using $V_{0}^{\mathrm{LO} \text { (wall) }}, V_{0}^{\mathrm{N}^{2} \mathrm{LO}}$, and $V_{0}^{\mathrm{N}^{2} \mathrm{LO}}+V_{2}^{\mathrm{N}^{2} \mathrm{LO}} \nabla^{2}$ are summarized in table 3 . The results from the different potentials are consistent with each other at low energies within statistical errors and the $\mathrm{N}^{2} \mathrm{LO}$ correction remains to be small (at most $\sim 1 \%$ difference) even for higher energies. These results are in line with the observation that the LO analysis from the wall source gives the correct phase shifts at low energies and the $\mathrm{N}^{2} \mathrm{LO}$ analysis gives only small correction even at higher energies [20].

\begin{tabular}{|c|ccc|}
\hline$\Delta E_{n}[\mathrm{MeV}]$ & $V_{0}^{\mathrm{LO}(\text { wall })}$ & $V_{0}^{\mathrm{N}^{2} \mathrm{LO}}$ & $V_{0}^{\mathrm{N}^{2} \mathrm{LO}}+V_{2}^{\mathrm{N}^{2} \mathrm{LO}} \nabla^{2}$ \\
\hline$n=0$ & $-1.5(0.3)$ & $-1.4(0.3)$ & $-1.4(0.3)$ \\
$n=1$ & $28.4(0.3)$ & $28.6(0.3)$ & $28.7(0.3)$ \\
$n=2$ & $60.4(0.4)$ & $60.8(0.4)$ & $61.2(0.4)$ \\
$n=3$ & $93.2(0.4)$ & $93.4(0.4)$ & $93.7(0.4)$ \\
$n=4$ & $124.1(0.3)$ & $124.3(0.3)$ & $124.6(0.3)$ \\
$n=5$ & $155.8(0.3)$ & $156.5(0.3)$ & $157.7(0.4)$ \\
$n=6$ & $186.5(0.3)$ & $187.3(0.3)$ & $189.1(0.4)$ \\
\hline
\end{tabular}

Table 3. The finite volume spectra using $V_{0}^{\mathrm{LO}(\text { wall })}, V_{0}^{\mathrm{N}^{2} \mathrm{LO}}$, and $V_{0}^{\mathrm{N}^{2} \mathrm{LO}}+V_{2}^{\mathrm{N}^{2} \mathrm{LO}} \nabla^{2}$ at $L=64$ evaluated at $t / a=13$. 


\section{B Eigenfunctions on various volumes}

Figure 13 shows the shapes of eigenfunctions on various volume.
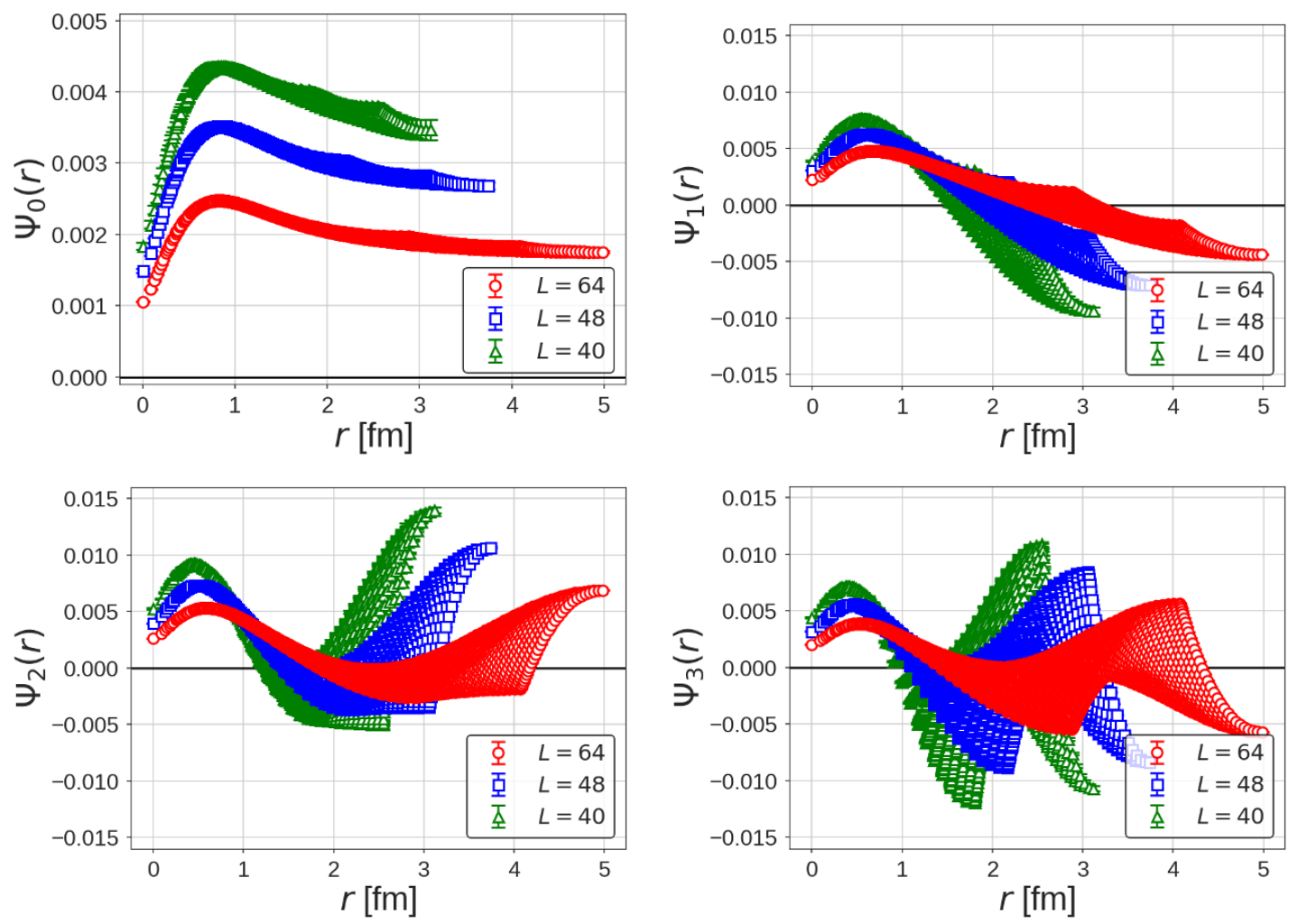

Figure 13. The low-lying eigenfunctions $\Psi_{n}(\vec{r})$ in the $A_{1}^{+}$representation of the HAL QCD Hamiltonian with $V_{0}^{\mathrm{LO} \text { (wall) }}$ at $t / a=13$ for $L=40,48$ and 64 . The top-left, top-right, bottom-left, bottom-right figure corresponds to $n=0,1,2,3$, respectively. The eigenfunction is normalized as $\sum_{\vec{r}}\left|\Psi_{n}(\vec{r})\right|^{2}=1$ and its sign is fixed to satisfy $\Psi_{n}(\overrightarrow{0})>0$.

\section{Reconstruction of the $R$-correlator}

In this appendix, we study how good the original $R$-correlator $R(\vec{r}, t)$ is approximated by the reconstructed $R$-correlator $\bar{R}(\vec{r}, t)$ given by

$$
\bar{R}(\vec{r}, t)=\sum_{n=0}^{n_{\max }} a_{n} \Psi_{n}(r) e^{-\Delta E_{n} t},
$$

where $n_{\max }=3,4,6$ for $L=40,48,64$, corresponding to the number of excited elastic states below the inelastic threshold. Figure 14 shows the comparison between the original (red squares) and the reconstructed (blue circles) $R$-correlators for the wall source (left) and the smeared source (right) at $t / a=13$. Shown together by black diamonds are the difference between the two, $R(\vec{r}, t)-\bar{R}(\vec{r}, t)$. To estimate the magnitude of the difference, 

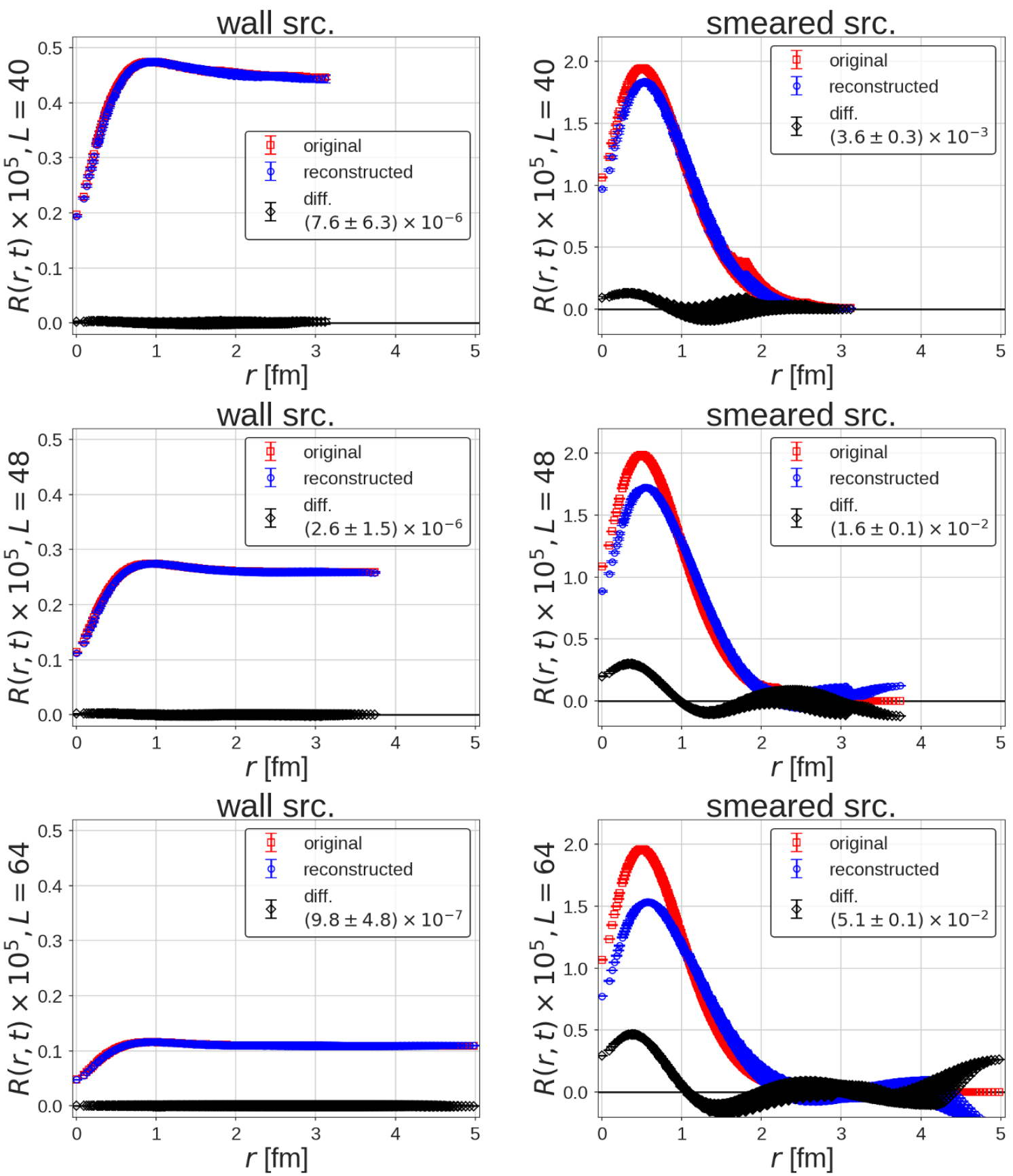

Figure 14. Comparisons between the original $R$-correlator (red squares) and the reconstructed $R$-correlator using low-lying eigenstates below the inelastic threshold (blue circles) at $t / a=13$ for $L=40$ (top), 48 (middle), 64 (bottom). The differences between the two $R$-correlators are shown by black diamonds and the values for the corresponding residual norms are given in legend panels. (Left) The wall source. (Right) The smeared source. 
we define the residual norm by

$$
\frac{\sum_{\vec{r}}|R(\vec{r}, t)-\bar{R}(\vec{r}, t)|^{2}}{\sum_{\vec{r}}|R(\vec{r}, t)|^{2}},
$$

whose values are also shown in the panels in figure 14 .

In the case of the wall source, it is found that the reconstruction works very well and the residuals are negligible $\left(\lesssim 10^{-5}\right)$. In the case of the smeared source, while the reconstruction is not as perfect as in the case of the wall source, the reconstructed $R$ correlator well reproduces the overall behavior of the original one where the residuals are only $\sim 0.4-5 \%$. The main origin of the residuals is the small discrepancies at short distances which become more apparent for a larger volume. This indicates that there remain small contributions from excited states above inelastic threshold in the case of the smeared source. In fact, since all elastic states couple to the smeared source with the same order of magnitude (see figure 7), there could remain contributions from elastic as well as inelastic states above the threshold for $R(\vec{r}, t)$ even at $t / a=13$, whose magnitude is expected to be larger for a larger volume due to the larger density of states.

\section{Reconstructed effective energy shifts}

We collect the results on the reconstructed effective energy shifts, eq. (4.6).

\section{D.1 The results on various volumes}

As shown in figure 15, we observe that the reconstruction generally works well. A small deviation at early time slices on $L=64$ for the smeared source is most likely due to the statistical fluctuations and/or the systematics due to contaminations from the states above the threshold discussed in appendix $\mathrm{C}$.

\section{D.2 Contributions from excited states to the effective energy shifts}

We study how each elastic excited state contributes to the effective energy shift for the smeared source, by changing the number of elastic excited states used in the reconstruction $\left(n_{\max }\right)$ in eq. (4.6).

Figure 16 shows the $n_{\max }$ dependence of the reconstructed effective energy shifts for the smeared source at $L=40,48$ and 64 , which are compared with the lowest eigenenergies $\Delta E_{0}$ (red bands). Due to the negative sign of $b_{n} / b_{0}<0$ (see figure 9 (right)), the reconstructed effective energy shifts are smaller than $\Delta E_{0}$. For $L=40$ and 48 , the dominant contribution comes from the first excited state $\left(n_{\max }=1\right)$ besides the ground state and higher modes give only minor corrections, while the second excited state $\left(n_{\max }=2\right)$ also gives significant contribution for $L=64$. These results indicate that the pseudoplateau structures around $t / a \sim 15$ are originated mostly from the scattering states below $\sim 90 \mathrm{MeV}$ (see table 2). 

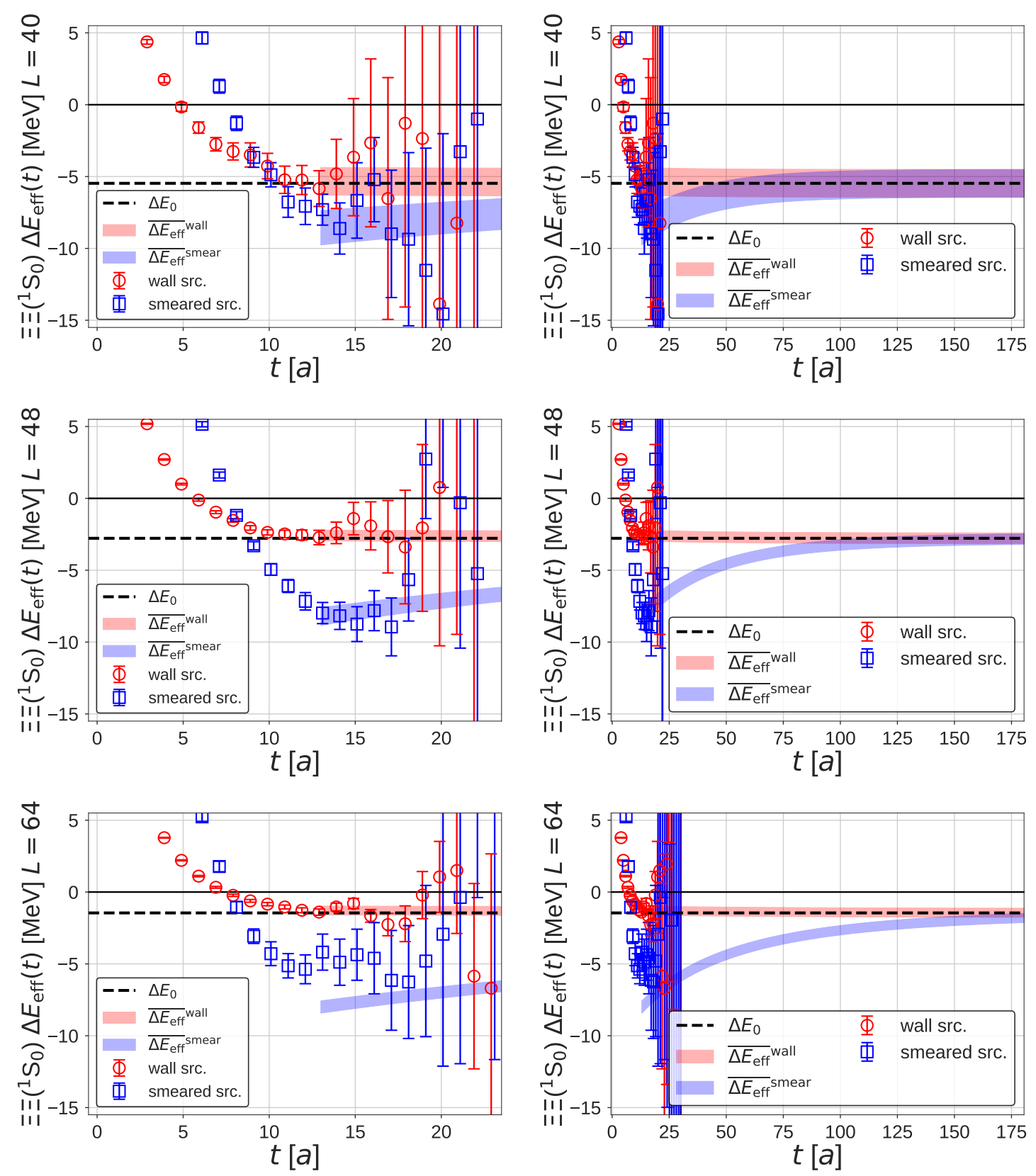

Figure 15. The reconstructed effective energy shifts $\overline{\Delta E_{\text {eff }}}\left(t, t_{0}=13 a\right)$ for the wall source (red bands) and the smeared source (blue bands) at $L=40,48$ and 64 . The effective energy shifts in the direct method are also shown for the wall (red circles) and smeared (blue squares) sources. The black dashed lines are the energy shifts for the ground state of the HAL QCD Hamiltonian in the finite volume evaluated at $t_{0} / a=13$. (Left) $0 \leq t / a \leq 24$. (Right) $0 \leq t / a \leq 175$. 

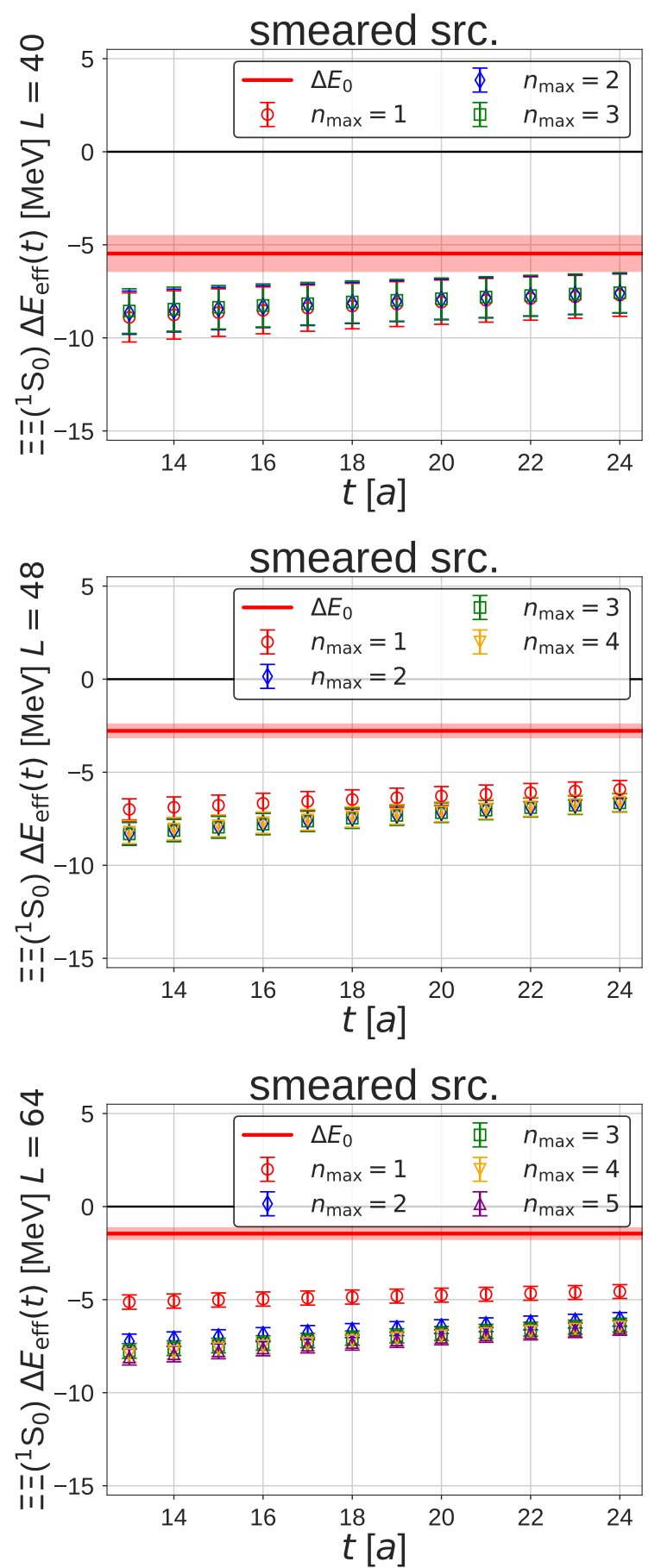

Figure 16. The mode number $\left(n_{\max }\right)$ dependence of the reconstructed effective energy shift $\overline{\Delta E_{\text {eff }}}\left(t, t_{0}=13 a\right)$ for the smeared source at $L=40$ (top), 48 (middle) and 64 (bottom). 


\section{E Effective energy shifts from the improved sink operator based on eigenfunctions}

Figure 17 shows effective energy shifts on various volumes, which are obtained from the improved two-baryon sink operator, projected to the ground state/first excited state in eq. (4.7). Results are found to be consistent with the corresponding finite-volume eigenenergies of the HAL QCD Hamiltonian. Small discrepancies for the smeared source on $L=64$ are most likely due to the statistical fluctuations and/or the systematics due to contaminations from the states above the threshold discussed in appendix C. Shown together in the left figure are the effective energy shifts in the direct method (without projection), where the significant deviation is observed for the smeared source.
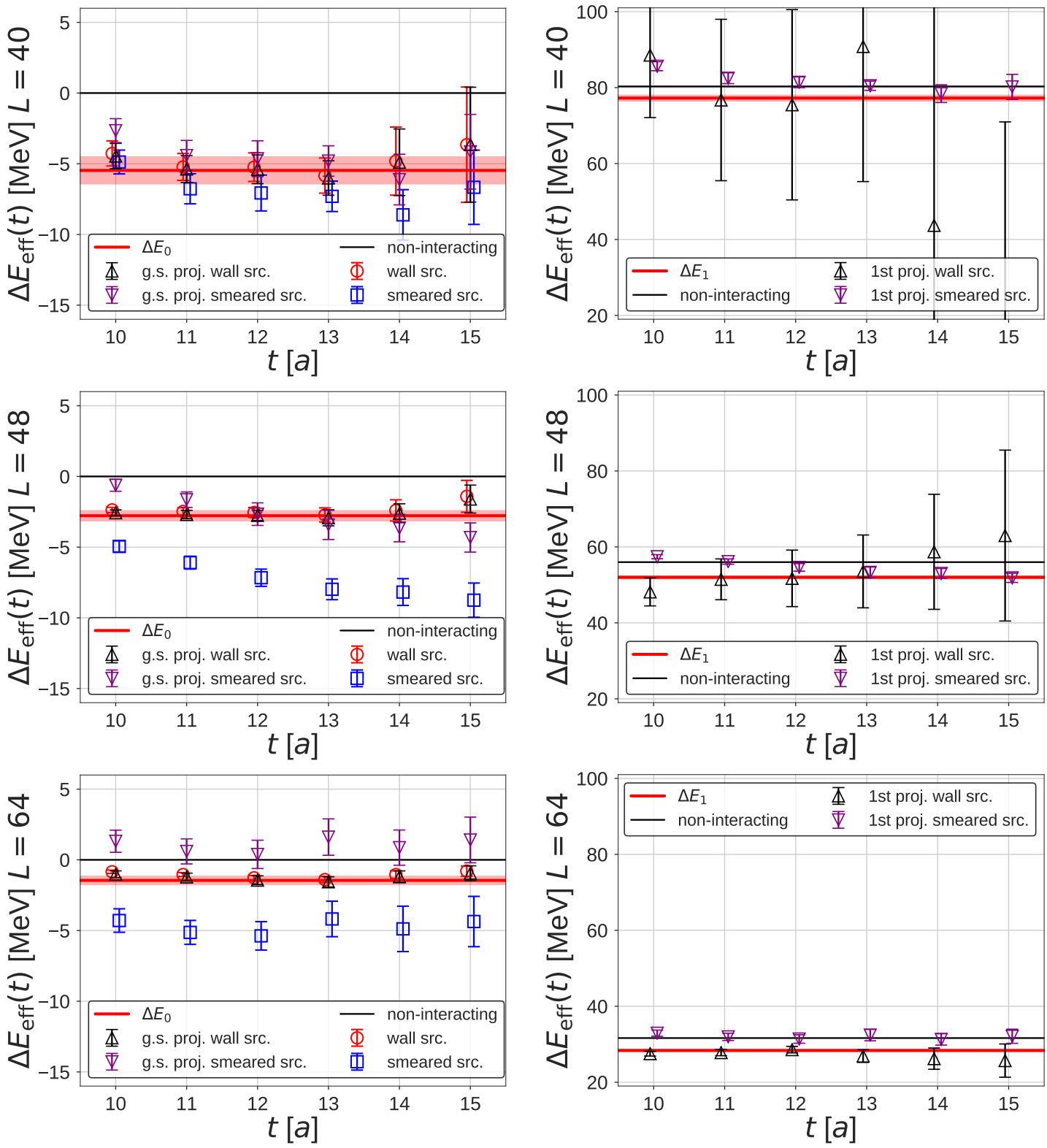

Figure 17. Same as figure 12 on $L=40$ (top), 48 (middle) and 64 (bottom). 
Open Access. This article is distributed under the terms of the Creative Commons Attribution License (CC-BY 4.0), which permits any use, distribution and reproduction in any medium, provided the original author(s) and source are credited.

\section{References}

[1] HAL QCD collaboration, T. Iritani et al., Mirage in Temporal Correlation functions for Baryon-Baryon Interactions in Lattice QCD, JHEP 10 (2016) 101 [arXiv:1607.06371] [INSPIRE].

[2] HAL QCD collaboration, T. Iritani et al., Are two nucleons bound in lattice QCD for heavy quark masses? Consistency check with Lüscher's finite volume formula, Phys. Rev. D 96 (2017) 034521 [arXiv: 1703.07210] [INSPIRE].

[3] T. Yamazaki, K.-i. Ishikawa, Y. Kuramashi and A. Ukawa, Study of quark mass dependence of binding energy for light nuclei in 2+1 flavor lattice QCD, Phys. Rev. D 92 (2015) 014501 [arXiv: 1502.04182] [INSPIRE].

[4] NPLQCD collaboration, M.L. Wagman et al., Baryon-Baryon Interactions and Spin-Flavor Symmetry from Lattice Quantum Chromodynamics, Phys. Rev. D 96 (2017) 114510 [arXiv: 1706.06550] [INSPIRE].

[5] Callat collaboration, E. Berkowitz et al., Two-Nucleon Higher Partial-Wave Scattering from Lattice QCD, Phys. Lett. B 765 (2017) 285 [arXiv:1508.00886] [INSPIRE].

[6] M. Lüscher, Volume Dependence of the Energy Spectrum in Massive Quantum Field Theories. 1. Stable Particle States, Commun. Math. Phys. 104 (1986) 177 [inSPIRE].

[7] M. Lüscher, Volume Dependence of the Energy Spectrum in Massive Quantum Field Theories. 2. Scattering States, Commun. Math. Phys. 105 (1986) 153 [INSPIRE].

[8] M. Lüscher, Two particle states on a torus and their relation to the scattering matrix, Nucl. Phys. B 354 (1991) 531 [inSPIRE].

[9] N. Ishii, S. Aoki and T. Hatsuda, The Nuclear Force from Lattice QCD, Phys. Rev. Lett. 99 (2007) 022001 [nucl-th/0611096] [INSPIRE].

[10] S. Aoki, T. Hatsuda and N. Ishii, Theoretical Foundation of the Nuclear Force in QCD and its applications to Central and Tensor Forces in Quenched Lattice QCD Simulations, Prog. Theor. Phys. 123 (2010) 89 [arXiv:0909.5585] [INSPIRE].

[11] HAL QCD collaboration, N. Ishii et al., Hadron-hadron interactions from imaginary-time Nambu-Bethe-Salpeter wave function on the lattice, Phys. Lett. B 712 (2012) 437 [arXiv: 1203.3642] [INSPIRE].

[12] HAL QCD collaboration, S. Aoki et al., Lattice QCD approach to Nuclear Physics, PTEP 2012 (2012) 01A105 [arXiv: 1206 .5088] [INSPIRE].

[13] S. Aoki, Lattice QCD and Nuclear Physics, in Modern perspectives in lattice QCD: Quantum field theory and high performance computing. Proceedings, International School, 93rd Session, Les Houches, France, August 3-28, 2009, pp. 591-628 (2010) [arXiv: 1008.4427] [INSPIRE].

[14] NPLQCD collaboration, S.R. Beane et al., Light Nuclei and Hypernuclei from Quantum Chromodynamics in the Limit of SU(3) Flavor Symmetry, Phys. Rev. D 87 (2013) 034506 [arXiv:1206.5219] [INSPIRE]. 
[15] HAL QCD collaboration, T. Inoue et al., Two-Baryon Potentials and H-Dibaryon from 3-flavor Lattice QCD Simulations, Nucl. Phys. A 881 (2012) 28 [arXiv:1112.5926] [INSPIRE].

[16] A. Francis, J.R. Green, P.M. Junnarkar, C. Miao, T.D. Rae and H. Wittig, Lattice QCD study of the $H$ dibaryon using hexaquark and two-baryon interpolators, arXiv: 1805.03966 [INSPIRE].

[17] T. Iritani for HAL QCD collaboration, Baryon interactions in lattice QCD: the direct method vs. the HAL QCD potential method, PoS (LATTICE2016) 107 (2016) [arXiv: 1610.09779] [INSPIRE].

[18] S. Aoki, T. Doi and T. Iritani, Sanity check for $N N$ bound states in lattice QCD with Lüscher's finite volume formula - Disclosing Symptoms of Fake Plateaux -, EPJ Web Conf. 175 (2018) 05006 [arXiv: 1707.08800] [INSPIRE].

[19] T. Iritani for HAL QCD collaboration, Two-baryon systems from HAL QCD method and the mirage in the temporal correlation of the direct method, EPJ Web Conf. 175 (2018) 05008 [arXiv: 1710.06147] [INSPIRE].

[20] HAL QCD collaboration, T. Iritani et al., Systematics of the HAL QCD Potential at Low Energies in Lattice QCD, Phys. Rev. D 99 (2019) 014514 [arXiv:1805.02365] [INSPIRE].

[21] G. Parisi, The Strategy for Computing the Hadronic Mass Spectrum, Phys. Rept. 103 (1984) 203 [INSPIRE].

[22] G.P. Lepage, The Analysis of Algorithms for Lattice Field Theory, in From Actions to Answers: Proceedings of the TASI 1989, T. Degrand and D. Toussaint eds., World Scientific, Singapore (1990) [CLNS-89-971] [INSPIRE].

[23] https://en.wikipedia.org/wiki/Sanity_check.

[24] K. Murano, N. Ishii, S. Aoki and T. Hatsuda, Nucleon-Nucleon Potential and its Non-locality in Lattice QCD, Prog. Theor. Phys. 125 (2011) 1225 [arXiv:1103.0619] [InSPIRE].

[25] K. Nishijima, Formulation of Field Theories of Composite Particles, Phys. Rev. 111 (1958) 995 [INSPIRE].

[26] W. Zimmermann, On the bound state problem in quantum field theory, Nuovo Cim. 10 (1958) 597 [INSPIRE].

[27] R. Haag, Quantum field theories with composite particles and asymptotic conditions, Phys. Rev. 112 (1958) 669 [INSPIRE].

[28] T. Yamazaki, K.-i. Ishikawa, Y. Kuramashi and A. Ukawa, Helium nuclei, deuteron and dineutron in 2+1 flavor lattice QCD, Phys. Rev. D 86 (2012) 074514 [arXiv:1207.4277] [INSPIRE].

[29] T. Doi and M.G. Endres, Unified contraction algorithm for multi-baryon correlators on the lattice, Comput. Phys. Commun. 184 (2013) 117 [arXiv: 1205.0585] [INSPIRE].

[30] NPLQCD collaboration, K. Orginos et al., Two nucleon systems at $m_{\pi} \sim 450 \mathrm{MeV}$ from lattice QCD, Phys. Rev. D 92 (2015) 114512 [arXiv:1508.07583] [INSPIRE].

[31] NPLQCD collaboration, S.R. Beane et al., Comment on "Are two nucleons bound in lattice QCD for heavy quark masses? - Sanity check with Lüscher's finite volume formula -", arXiv:1705.09239 [INSPIRE]. 
[32] M. Lüscher and U. Wolff, How to Calculate the Elastic Scattering Matrix in Two-dimensional Quantum Field Theories by Numerical Simulation, Nucl. Phys. B 339 (1990) 222 [InSPIRE].

[33] R.A. Briceno, J.J. Dudek and R.D. Young, Scattering processes and resonances from lattice QCD, Rev. Mod. Phys. 90 (2018) 025001 [arXiv:1706.06223] [InSPIRE].

[34] HAL QCD collaboration, S. Gongyo et al., Most Strange Dibaryon from Lattice QCD, Phys. Rev. Lett. 120 (2018) 212001 [arXiv:1709.00654] [INSPIRE].

[35] HAL QCD collaboration, T. Iritani et al., $N \Omega$ dibaryon from lattice $Q C D$ near the physical point, arXiv:1810.03416 [INSPIRE].

[36] HAL QCD collaboration, T. Inoue et al., Strange Nuclear Physics from QCD on Lattice, in 13th International Conference on Hypernuclear and Strange Particle Physics (HYP 2018), Portsmouth Virginia, U.S.A., June 24-29, 2018 [arXiv: 1809. 08932] [INSPIRE].

[37] T. Amagasa et al., Sharing lattice QCD data over a widely distributed file system, J. Phys. Conf. Ser. 664 (2015) 042058 [INSPIRE].

[38] http://www.lqcd.org/ildg.

[39] http://www.jldg.org.

[40] Columbia Physics System (CPS), http://usqcd-software.github.io/CPS.html.

[41] Bridge++, http://bridge.kek.jp/Lattice-code/.

[42] M. Schröck and H. Vogt, Coulomb, Landau and Maximally Abelian Gauge Fixing in Lattice QCD with Multi-GPUs, Comput. Phys. Commun. 184 (2013) 1907 [arXiv:1212.5221] [INSPIRE].

[43] T. Boku et al., Multi-block/multi-core SSOR preconditioner for the QCD quark solver for $K$ computer, PoS (LATTICE2012) 188 (2012) [arXiv:1210.7398] [INSPIRE].

[44] M. Terai et al., Performance Tuning of a Lattice QCD code on a node of the K computer (in Japanese), IPSJ Trans. Adv. Comput. Syst. 6 (2013) 43. 